\title{
Understanding PPA-Completeness
}

\author{
Xiaotie Deng ${ }^{1}$, Jack R. Edmonds ${ }^{2}$, Zhe Feng ${ }^{3}$, Zhengyang Liu ${ }^{1}$, \\ $\mathrm{Qi} \mathrm{Qi}^{5}$, and Zeying $\mathrm{Xu}^{6}$
}

1 Department of Computer Science, Shanghai Jiao Tong University, Shanghai, China

dengxiaotie@sjtu.edu.cn

2 Waterloo, Canada

jack.n2m2m6@gmail.com

3 Zhiyuan College, Shanghai Jiao Tong University, Shanghai, China

sjtufz@sjtu.edu.cn

4 Department of Computer Science, Shanghai Jiao Tong University, Shanghai, China

lzy5118@sjtu.edu.cn

5 Department of IELM, Hong Kong University of Science and Technology,

Hong Kong

kaylaqi@ust.hk

6 Department of Mathematics, Shanghai Jiao Tong University, Shanghai, China zane_xu@sjtu.edu.cn

\begin{abstract}
We consider the problem of finding a fully colored base triangle on the 2-dimensional Möbius band under the standard boundary condition, proving it to be PPA-complete. The proof is based on a construction for the DPZP problem, that of finding a zero point under a discrete version of continuity condition. It further derives PPA-completeness for versions on the Möbius band of other related discrete fixed point type problems, and a special version of the Tucker problem, finding an edge such that if the value of one end vertex is $x$, the other is $-x$, given a special anti-symmetry boundary condition.

More generally, this applies to other non-orientable spaces, including the projective plane and the Klein bottle. However, since those models have a closed boundary, we rely on a version of the PPA that states it as to find another fixed point giving a fixed point. This model also makes it presentationally simple for an extension to a high dimensional discrete fixed point problem on a non-orientable (nearly) hyper-grid with a constant side length.
\end{abstract}

1998 ACM Subject Classification F.1.3 Complexity Measures and Classes

Keywords and phrases Fixed Point Computation, PPA-Completeness

Digital Object Identifier 10.4230/LIPIcs.CCC.2016.23

\section{Introduction}

In his seminal work on understanding the time complexity of the parity argument, Papadimitriou introduced the now well known class PPAD [27] that has influenced a generation of algorithmic game theorists in their study of economic computations. In the same paper, Papadimitriou also defined a more inclusive complexity class PPA (Polynomial Parity Argument) of search problems whose solution is guaranteed to exist through a proof based on the fact that "Any undirected graph with an odd-degree vertex must have another one". In contrast to PPA, PPAD is based on another straightforward principle: "Any directed graph that has an unbalanced node must have another".

cc) (i) Xiaotie Deng, Jack R. Edmonds, Zhe Feng, Zhengyang Liu, Qi Qi and Zeying $\mathrm{Xu}$;

licensed under Creative Commons License CC-BY

31st Conference on Computational Complexity (CCC 2016).

Editor: Ran Raz; Article No. 23; pp. 23:1-23:25

Leibniz International Proceedings in Informatics

LIPICS Schloss Dagstuhl - Leibniz-Zentrum für Informatik, Dagstuhl Publishing, Germany 
The class PPA is a superset of PPAD, and the intuitive reason is that directions are helpful: Finding another node of the appropriate kind is harder to solve when there are no directions; in fact, oracle separation is known [3]. This difference has also reflected in our understanding in the two classes, especially with regard to their complete problems. The class PPAD has now many problems that have been shown complete for PPAD such as in the incomplete list of 25 of them [22] gathered by Kintali. The class PPA-complete, however, did not fare as well.

On the one hand, there are many interesting existence theorems in Graph Theory, Combinatorics and Number Theory for which the computational problems are in PPA [27]: Smith's theorem [30] and related existentially polytime (graph) theorems [5], Chevalley's theorem [10] and Alon's Combinatorial Nullstellensatz [2], among others. Remarkably, the problem of factoring an integer has been recently proved to belong to PPA (via randomized reductions) [21], and the inclusion of this fundamental and critical problem gives the class a new significance.

On the other hand, we know few PPA-complete problems besides the generic one, unfortunately. The only exceptions are certain versions of Sperner's problem for rather esoteric non-orientable bodies. About ten years after the introduction of the class, Grigni [17] had the important idea that the right geometric context for PPA are non-orientable bodies, and showed that a version of the Sperner problem in the non-orientable three dimensional space is complete in the class. Soon after, Friedl et al. [15] strengthened it to a non-orientable and locally two-dimensional orientable space.

In general, it would be nice to have a growing strong collection of PPA-complete problems (like we have for PPAD), which with luck could eventually include factoring. The progress has been slow: another ten years passed without any progress in our understanding of the class PPA-complete for this problem many scientists are interested in.

\section{Contributions}

Our main results first end the quest for a complete fixed point characterization of the PPA-complete class. It provides a sharp division on what can be done and what cannot be done in computing different versions of the fixed point problem on the Möbius band. In particular, it does so by completing the task started by Friedl et al. [15], to reduce the next dimension demanded by the seminal result of Grigni [17], with the help of a technique developed by Chen and Deng [7], on the 2D Möbius version of a zero point problem, referred to as DPZP and conceptualised in $[20,8,7,11]$. Together with the results of Grigni and Friedl, et al., they raise a theoretical connection of computational complexity to topology. The comparison between the $2 \mathrm{D}$ versions makes a strong case for this distinction.

Next, as the past works of Chen and Deng [7] as well as Deng, Qi, Saberi and Zhang [11] unify the complexity of the various discrete fixed point concepts in principle the above result implies that the same result holds for all the related discrete fixed points on the Möbius band. However, this may not always hold in general. We develop a new reduction approach to derive those results on the Möbius band. In particular, the 2D Tucker on orientable space were proven PPAD-hard, originally in the first principle by Pálvölgyi [26] and then by reduction to another discrete fixed point [11]. Both approaches are complicated where applied to the Möbius version. Our new reduction approach makes it easy to be shown in both ways of containing and contained in the PPA class. The same holds for the other discrete fixed point problems.

Third, the simplicity of our 2D version has been handy to make further applications. On the higher constant dimension non-orientable space, all the discrete fixed point problems follow from the $2 \mathrm{D}$ results to become PPA-complete. Those cannot be easily obtained from 
the past works for the Sperner problem alone. An even bigger challenge here is whether the PPAD-completeness of the constant side length higher dimensional Sperner's problem developed by Chen, et al., [9], can be extended to the non-orientable space. Using a new (dicephalic snake) embedding lemma, together with a few demanding technical details, a 2D Sperner version is used to reduce to the higher dimension and constant side length Sperner problem on non-orientable space, and to prove the PPA-hardness of the latter. The proof involves quite some technical details but still accessible, which would be extremely difficult due to the subtlety of the boundary conditions of the non-orientable case if our Sperner on the 2D Möbius band is constructed differently. The same subtlety applies to the other discrete fixed point versions.

Fourth, the concept of the index, with modification of mod 2, is helpful both for the proofs that the above problems are in PPA, It has also be applied to develop algorithmic solutions for the oracle model of the computational problem. This approach had delivered the matching algorithmic bound for the oracle models for the fixed point problem in the orientable space [8], closing a previously almost tight gap [19]. The extension to the non-orientable space is quite natural by simply taking a mod 2 operation upon that for the orientable space. But it proves very effective. In comparison, past works have taken the path following paradigm for the fixed point computation. There are some subtleties in using index for the non-orientable space. We should not interpret the index and other values in the definitions as in the orientable space: the sense of direction no longer holds in non-orientable space at least in one dimension. Even though they are named similarly, we still need to treat them differently.

Fifth, the techniques for the 3D version may bear some similarity with our 2D version, it is exactly the articulation or, the simplicity if one prefers, in the 2 dimensional results that allows better applications to even better understanding in related problems. As we prove related results for other non-orientable spaces such as the Klein bottle or projective space, we would have to refer to less natural 3D (in 5 dimensions) Klein solid bottle or $3 \mathrm{D}$ projective space, with unbearable complications in the proofs. One such case is in the beautiful PSPACE proof of the other end of the line for the path following algorithm in the $2 \mathrm{D}$ discrete fixed point proof by Goldberg[16]. In addition, we had 20 years after the definition of PPA by Papadimitriou, 15 years after Grigni's 3D non-orientable space Sperner's PPA-completeness, and 10 years after the locally 2D Sperner's PPA-completeness by Friedl, et al. [15]. It is a time for a better understanding.

\section{Relevance of the Möbius Band}

The stories of the Möbius band have been a curiosity out of the Mind, such as a brain's toy of German mathematicians August Ferdinand Möbius (and Johann Benedict Listing), and the fascination art in the parade of ants by a Dutch artist M.C. Escher [13]. In recent years, it becomes a possibility in scientific discoveries. Scientists made assembled object created by nano technology [18], proposed technical tool to develop negative refractive index materials [14], made experimental observation in electromagnetic metamaterial systems [6]. In our work, it plays a role in understanding theoretical complexity of PPA-completeness. Hopefully, one day, they will become truly useful like other creatures of human imagination, if one so demands.

\section{Related Literatures}

The standard Sperner's problem, 3D-Sperner, is among the first natural problem proved to be PPAD-complete by Papadimitriou [27]. The problem 2D-Sperner is proved to be 
PPAD-complete by Chen and Deng [7]. In [17], Grigni proposed the brilliant idea using non-orientable space to model the 3D-Sperner as a PPA-complete problem. The only other known PPA-complete problem is the Sperner problem on a sophisticated locally 2D structure by Friedl, Ivanyos, Santha and Verhoeven [15].

Lemke-Howson's algorithm [24] for Nash equilibrium computation has started a path following paradigm. However, a worst case exponential lower bound was known for this algorithm by Savani and von Stengel [28]. It was shown that the other PPAD-complete problems demand, under the oracle model, exponential time including the fixed point problem by Hirsch, Papadimitriou and Vavasis [19]. It was further shown to have a tight exponential time by Chen and Deng [8], which was extended to include several discrete versions of the fixed point problem by Deng, Qi, Saberi and Zhang [11].

For the PPA class, the path following method was known to take an exponential time for the Smith problem by Krawczyk [23, 4]. It has been extended to related problems, such as an exponential time bound for finding the second perfect matching on Eulerian graphs by Edmonds and Sanita [12]. An extensive discussion on related problems can be found in [5]. Subsequently, Aisenberg et. al. [1] improved our result - proving that general version for 2D-Tucker is PPA-complete using an elegant trick.

\section{Organization of Presentation}

We prove that the natural Möbius band versions of the problems, Sperner, DPZP and Tucker to be PPA-complete. A neat reduction allows the problem of finding one fixed point be extended to given-one-find-another types of PPA problems. Along with several important technical details, a dicephalic snake lemma is crucial for the padding and folding to create a higher dimensional fixed point on a non-orientable grid in order to reduce the problem to one of constant side lengths.

The paper is laid out as follows: In Section 2, we will show some necessary definitions and notations. In Section 3, we show a key result, the proof of PPA-completeness of the problem mn-DPZP and its applications. In Section 4, we extend our work to prove a high-dimensional non-orientable version of fixed point. In Section 5, we applies our main result to other non-orientable spaces, including the projective plane and the Klein bottle. We prove the PPA-completeness of the problem of finding another fixed point on the projective plane and on the Klein bottle. In Section 6, we discuss the generality of the results obtained here in related settings. Finally we discuss potential future works. Because of the space limitation, we put most of our proofs in the Appendix.

\section{Preliminaries and Definitions}

PPA, (in its complete form, the Polynomial Parity Argument class), is a class of search problems based on an exponential size graph consisting of nodes of maximum degree two, with a given node of degree one. The problem asks for an output of another node of degree one, which is guaranteed to exist by the parity argument. More formally, we define it by a complete problem, named AEUL as follows:

- Definition 2.1 (Another End of Undirected Lines). Given an input circuit $T_{n}$ of polynomial size in $n$ which takes as input $u$ in the configuration space $C_{n}=\{0,1\}^{n}$, returns as output $T_{n}(u)$ in the form $\langle v, w\rangle,\langle v\rangle$, or \langle\rangle where $v>w$ and $v, w \in C_{n} \backslash\{u\} . \mathbf{0}^{n}$ is a given configuration of one tuple, i.e., $\left|T_{n}\left(\mathbf{0}^{n}\right)\right|=1$. The search problem is to find another configuration $v, v \neq \mathbf{0}^{n}$ such that $\left|T_{n}(v)\right|=1$. We should write it as AEUL for short. 


\section{Möbius Band}

It is obtained from a rectangle by merging its left and right sides after twisting it 180 degrees (counter)-clockwise to form a one-boundary and one-surface band. Therefore, it is non-orientable. More formally,

Definition 2.2 (Möbius Band). Let $V_{N, M}=\left\{\mathbf{p}=\left(p_{1}, p_{2}\right) \in \mathbb{Z}^{2}:-N \leq p_{1} \leq N,-M \leq\right.$ $\left.p_{2} \leq M\right\}$. A Möbius band is obtained by twisting $V_{N, M} 180$ degrees clockwise and then joining every vertex $(N, y)$ with $(-N,-y)$ to form a loop. We denote it by $B_{N, M}$. A function $f$ is defined on the Möbius band $B_{N, M}$ iff $\forall y:-M \leq y \leq M$, we have $f((N, y))=f((-N,-y))$ on $V_{N, M}$.

- Definition 2.3 (Standard Triangulation). For each $i, j \in \mathbb{Z}:-N \leq i<N,-M \leq j<M$, we link $(i, j)$ with $(i+1, j+1)$ on the grids $V_{N, M}$ and $B_{N, M}$.

We call every unit square in the standard triangulated grid $V_{N, M}$ a base square, every unit side length triangle of it a base triangle, its every edge a base edge.

\section{Index}

We now define the index $[29,31]$ but adopt it for the non-orientable space $B_{N, M}$.

Consider a coloring by $\{0,1,2\}$ of vertices in $B_{N, M}$, one vertex is assigned by one color. If a base triangle $\delta$ has all three colors, we define its index as 1 . Otherwise, the index is 0 . Alternatively, we define an edge index to be 1 if it is colored by both 1 and 2 . The index of a base triangle is the sum of indices of its three edges, mod 2. It prepares us to define the index on Möbius band.

Definition 2.4 (Index of a Non-orientable Triangulated Möbius Grid $B_{N, M}$ ). Given a triangulated Möbius grid $B_{N, M}$, a coloring $\phi: B_{N, M} \rightarrow\{0,1,2\}$ of its vertices. The index of $B_{N, M}$ is defined as

$$
\operatorname{index}\left(B_{N, M}, \phi\right):=\sum_{\delta \text { is a base triangle } \in B_{N, M}} \operatorname{index}(\delta, \phi) \quad(\bmod 2) .
$$

Immediately, one derive the following lemma about indices on the Möbius band.

\section{- Lemma 2.5.}

$$
\operatorname{index}\left(B_{N, M}, \phi\right)=\sum_{e \in \partial B_{N, M}} \operatorname{index}(e, \phi) \quad(\bmod 2),
$$

where $\partial B_{N, M}$ is the boundary of $B_{N, M}$, that is, $\partial B_{N, M}:=\left\{\left(p_{1}, \pm M\right) \in \mathbb{Z}^{2}:-N \leq p_{1} \leq N\right\}$.

\section{DPZP}

We should introduce several concepts to prepare its definition as a numeric version of the original direction preserving zero point.

- Definition 2.6 (Möbius Numeric Feasible Function). A function $f: B_{N, M} \rightarrow\{0, \pm 1, \pm 2\}$ is feasible if it satisfies the Möbius condition, $f((N, y))=f((-N,-y)), \forall y \in \mathbb{Z},-M \leq y \leq M$.

Definition 2.7 (Möbius Numeric Direction-preserving Function). A function $f: B_{N, M} \rightarrow$ $\{0, \pm 1, \pm 2\}$ is direction-preserving if for any $\mathbf{p}, \mathbf{q} \in B_{N, M}$ where $\|\mathbf{p}-\mathbf{q}\|_{\infty}=1$ and $f(\mathbf{p}) \neq 0$, we have $f(\mathbf{p})+f(\mathbf{q}) \neq 0$. 
- Definition 2.8 (Zero Point Base Triangle). Given a function $f: B_{N, M} \rightarrow\{0, \pm 1, \pm 2\}$, a base triangle $\delta$ of a triangulated Möbius Grid is called a zero point base triangle of $f$ if $\{f(\mathbf{p}): \mathbf{p} \in \delta\}=\{0,1,2\}$.

- Definition 2.9 (Admissible Boundary Condition). A function $F: B_{N, M} \rightarrow\{0, \pm 1, \pm 2\}$ is called admissible if it satisfied the following boundary conditions:

- $F((0, M))=-2 ; F((0,-M))=2$

- $F((i, M))=F((-i,-M))=-1$, for every $i \in \mathbb{Z}: 0<i \leq N$

- $F((-i, M))=F((i,-M))=1$, for every $i \in \mathbb{Z}: 0<i \leq N$

- Definition 2.10 (Numeric Möbius DPZP). Given as input, a triangulated Möbius Grid $B_{N, M}$, and a polynomial-time machine $F$, which generates a numeric direction-preserving feasible admissible function $f$ on $B_{N, M}: f(\mathbf{p}) \in\{0, \pm 1, \pm 2\}, \forall \mathbf{p} \in B_{N, M}$, we are required to output $\mathbf{p}: f(\mathbf{p})=0$.

As the function $F(\cdot, \cdot)$ for mn-DPZP has five values, the index defined above does not apply. We should introduce a new definition of index for mn-DPZP.

- Definition 2.11 (Index of a Base Edge and a Base Triangle in mn-DPZP). Given an mnDPZP grid $B_{N, M}$, a coloring $F: B_{N, M} \rightarrow\{0, \pm 1, \pm 2\}$, of its vertices. The index of an edge is 1 if the colors of its two end vertices are $\{1,2\}, 0$ otherwise. The index of a base triangle is the sum of the indices of its three edges $(\bmod 2)$.

- Definition 2.12 (Index of mn-DPZP). Given a mn-DPZP grid $B_{N, M}$, a coloring $F$ : $B_{N, M} \rightarrow\{0, \pm 1, \pm 2\}$, of its vertices. The index of $B_{N, M}$ is defined as

$$
\operatorname{index}\left(B_{N, M}, F\right):=\sum_{\delta \text { is a base triangle } \in B_{N, M}} \operatorname{index}(\delta, F) \quad(\bmod 2) .
$$

We have the following lemma on the Möbius grid.

- Lemma 2.13. index $(\delta, F)=1$ if and only if $F(\delta)=\{0,1,2\}$. Furthermore, index $\left(B_{N, M}, F\right)=$ $\sum_{e \in \partial B_{N, M}} \operatorname{index}(e, F)(\bmod 2)$, where $\partial B_{N, M}$ is the boundary of $B_{N, M}$.

Using the index on non-orientable surfaces, it is immediately that:

- Lemma 2.14. The Numeric Möbius DPZP with the admissible boundary always has a zero point. Finding a zero point is a PPA problem.

We should next list the results for other related discrete fixed point concepts. We call the problem of finding a fully colored base triangle on Möbius band $B_{N, M}$ the m-Sperner problem.

- Definition 2.15 (m-Sperner). Consider a triangulated Möbius grid $B_{N, M}$ and a polynomialtime machine $G$, which generates a function $g$ on $B_{N, M}: g(\mathbf{p})=G(\mathbf{p}) \in\{0,1,2\}, \forall \mathbf{p} \in B_{N, M}$. Further, we require that $g(\cdot)$ satisfies the m-Sperner boundary condition, defined as follows.

- $G((0, M))=0 ; G((0,-M))=2$

- $G((i, M))=G((-i,-M))=0$, for every $i \in \mathbb{Z}: 0<i \leq N$

- $G((-i, M))=G((i,-M))=1$, for every $i \in \mathbb{Z}: 0<i \leq N$

The required output is a base triangle which contains all three colors.

- Lemma 2.16. On any admissible triangulated Möbius band $B_{N, M}$ for an $m$-Sperner instance, the number of Sperner base triangles is odd. Finding one of those is in PPA. 
Proof. As m-Sperner has index 1, the oddness follows. The reduction to an AEUL is similar to the above for the mn-DPZP problem.

We define the simple Möbius version of Tucker as follows.

- Definition 2.17 (sm-Tucker). Consider a triangulated Möbius grid $B_{N, M}$ and a polynomialtime machine $G$, which generates a function $g$ on $B_{N, M}: g(\mathbf{p})=G(\mathbf{p}) \in\{ \pm 1, \pm 2\}, \forall \mathbf{p} \in B_{N, M}$. Further, we require that $g(\cdot)$ satisfies the special antipodal boundary condition, defined as follows:

- $g((0, M))=-2 ; g((0,-M))=2$

- $g((i, M))=g((-i,-M))=-1$, for every $i \in \mathbb{Z}: 0<i \leq N$

- $g((-i, M))=g((i,-M))=1$, for every $i \in \mathbb{Z}: 0<i \leq N$

and Möbius boundary condition which is $g((N, y))=g((-N,-y))$. The required output is a complementary edge.

- Lemma 2.18. On sm-Tucker, there is always a complementary edge. Finding one is in $P P A$.

Proof. Changing the colors $\{-1,-2\}$ of the vertices in sm-Tucker into 0 , we reduce the problem to $\mathrm{m}$-Sperner. As the boundary of the m-Sperner has index 1 , there is always a fully colored base triangle $\delta$. The vertex colored 0 in $\delta$ was originally either -1 or -2 in the sm-Tucker, we obtain a complementary edge in the sm-Tucker. The claims follow.

\section{PPA-completeness of mn-DPZP and Its Applications}

We have already proven that mn-DPZP is in PPA in the last section. We now prove the PPAhardness of the mn-DPZP. For any input to $A E U L\left(T_{n}, C_{n}, 0^{n}\right)$, we construct an mn-DPZP instance in polynomial time so that each zero point in the mn-DPZP instance maps back to an end vertex for some lines in the original instance of $A E U L\left(T_{n}, C_{n}, 0^{n}\right)$, and vice versa.

Our proof embeds the $\operatorname{AEUL}\left(T_{n}, C_{n}, 0^{n}\right)$ graph on the Möbius band. The reduction is motivated by the original proof of 2D Sperner being PPAD-complete by Chen and Deng [7].

Given a simple undirected graph $G=(V, E)$, let $|V|=N=2^{n}$, we define $G^{*}=\left(V^{*}, E^{*}\right)$, where $V^{*}=V_{12 N^{2}, 24 N}$, and $E^{*}=\left\{\left(\mathbf{p}, \mathbf{p}^{\prime}\right):\left\|\mathbf{p}-\mathbf{p}^{\prime}\right\|_{1}=1\right\}$, i.e., $\left(\mathbf{p}, \mathbf{p}^{\prime}\right)$ is an edge in $G^{*}$ if and only if their $L_{1}$ distance is 1 . For every $\mathbf{p} \in V^{*}$, let $K_{\mathbf{p}}=\left\{\mathbf{q}: q_{i} \in\left\{p_{i}, p_{i}+1\right\}\right\}, i=1,2$ to be the vertex set containing all 4 vertices in the base square having $\mathbf{p}$ at the left bottom corner, and $E_{\mathbf{p}}^{1}=\{\{\mathbf{p}, \mathbf{p}+(0,1)\},\{\mathbf{p}+(1,0), \mathbf{p}+(1,1)\}\}, E_{\mathbf{p}}^{2}=\{\{\mathbf{p}, \mathbf{p}+(1,0)\},\{\mathbf{p}+(0,1), \mathbf{p}+(1,1)\}\}$ to be its two subsets of edges of $K_{\mathbf{p}}$. For $\mathbf{p}, \mathbf{q} \in V^{*}$, if $p_{i}=q_{i}, i=1$ or 2 , let $\mathbf{u}^{1}, \mathbf{u}^{2}, \ldots, \mathbf{u}^{m} \in$ $\mathbb{Z}^{2}$ be all the integer internal points on segment pq which are labeled along pq, where $\mathbf{u}^{1}=\mathbf{p}$ and $\mathbf{u}^{m}=\mathbf{q}$. We say $K_{\mathbf{p}}$ and $K_{\mathbf{q}}$ are connected iff edges set $\cup_{k=1}^{m} E_{\mathbf{u}^{k}}^{i} \subseteq E^{*}$, we denote it by $K_{\mathbf{p}} K_{\mathbf{q}}$

On the Möbius band, we also allow that $K_{\left(12 N^{2}-1, y\right)}$ and $K_{\left(-12 N^{2},-y-1\right)},-24 N \leq$ $y \leq 24 N-1$ can be connected, that is $E_{\left(12 N^{2}-1, y\right)}^{2} \cup E_{\left(-12 N^{2},-y-1\right)}^{2} \subseteq E^{*}$. If we have $K_{\mathbf{u}^{1}} K_{\mathbf{u}^{2}}, K_{\mathbf{u}^{2}} K_{\mathbf{u}^{3}}, \ldots, K_{\mathbf{u}^{m-1}} K_{\mathbf{u}^{m}}$, but these points $\mathbf{u}^{1}, \mathbf{u}^{2}, \cdots, \mathbf{u}^{m}$ don't share the same xcoordinate nor y-coordinate, hence the edges introduced need to make turns in its directions to connect $u^{1}$ to $u^{m}$. We make a special note that, at a turn on $K_{u}$ toward the right-upper direction, the edges $\{\{u, u+(0,1)\},\{u+(0,1), u+(1,1)\}\}$ will be removed to make the nodes along the paths be of degree no more than two.

Intuitively, $G^{*}$ is a plannar embedding of the graph $G$ for AEUL with vertices $\{0,1, \ldots, N-$ $1\}$. The construction is motivated by and has some similar details to that of Chen and Deng [7]. Making it work on the Möbius band requires new ideas. 


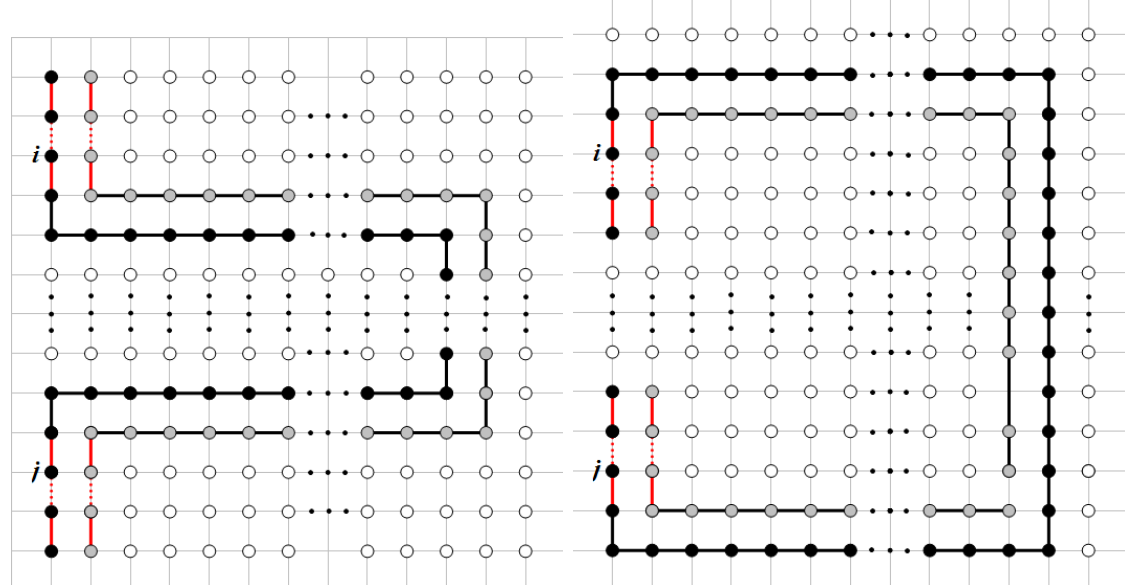

Figure 1 Connecting vertices.

For every $i: 0 \leq i<N$, vertex $i$ of $G$ maps to a vertex set $S_{i}=\cup_{k=24 i}^{24 i+11}\left\{K_{(0, k)}\right\}$. That is, we create a fixed-length "tube" $S_{i}$ for the vertex $i$ in $G$. We call it a "vertex tube". Every such tube has two ends, called up and down, dependent on their values of the second coordinates on $V^{*}$, denoted by $S_{i}^{u p}=K_{(0,24 i+11)}$ and $S_{i}^{\text {down }}=K_{(0,24 i)}$. We make a change in the embedding of the starting node $\mathbf{0}^{n}$ : for $i=0, S_{0}=\cup_{k=-24 N}^{(24 i+11)}\left\{K_{(0, k)}\right\}$ in $G^{*}$.

Edge $i j$ appears in $G$ iff there is an undirected path between one of $\left\{S_{i}^{\text {up }}, S_{i}^{\text {down }}\right\}$ and one of $\left\{S_{j}^{u p}, S_{j}^{d o w n}\right\}$. Let $i j \in E$ and $i k \in E$ be the two edges connected to $j$ and $k$ from $i$. If $j>k$ we call $j$ the bigger neighbour and $k$ the smaller neighbour of the vertex $i$.

For each vertex tube, we connect its up end to its bigger neighbour (if the degree of the vertex is 1 , we also take it as the bigger one), and its down end to the smaller neighbour (if any).

If $(i, j)$ is an edge in $G$, let $y_{i}, y_{j}$ be the y-coordinates of the ends of tube $i$ and $j$ where need to be linked together. Let $t=12(N \cdot \max \{i, j\}+\min \{i, j\})$. We consider two different connection cases:

1. $S_{i}^{\text {up }}-S_{j}^{\text {down }}$ or $S_{i}^{\text {down }}-S_{j}^{u p}$ : we add edges $K_{\left(0, y_{i}\right)} K_{\left(t, y_{i}\right)}, K_{\left(t, y_{i}\right)} K_{\left(t, y_{j}\right)}, K_{\left(t, y_{j}\right)} K_{\left(0, y_{j}\right)}$ into $E^{*}$.

2. $S_{i}^{u p}-S_{j}^{u p}$ or $S_{i}^{\text {down }}-S_{j}^{\text {down}}$ : w.l.o.g., we assume that $i<j$, we add edges $K_{\left(0, y_{i}\right)} K_{\left(12 N^{2}-1, y_{i}\right)}$, $K_{\left(12 N^{2}-1, y_{i}\right)} K_{\left(-12 N^{2},-y_{i}-1\right)}, K_{\left(-12 N^{2},-y_{i}-1\right)} K_{\left(-t-1,-y_{i}-1\right)}, K_{\left(-t-1,-y_{i}-1\right)} K_{\left(-t-1, y_{j}\right)}$ and $K_{\left(-t-1, y_{j}\right)} K_{\left(0, y_{j}\right)}$ into $E^{*}$.

Case 1 is illustrated in Figure 1, which is a normal case. The crucial difference that would involve in the Möbius band structure $B_{12 N^{2}, 24 N}$ is case 2, illustrated in Figure 2. For example, if degree of $i$ is 2 , i.e. $T(n, i)=\langle j, k\rangle, k>i, j$, also we assume that $i>j$ and $T(n, j)=\langle k, i\rangle$. Let $t=10(n \cdot i+j)$, we will link $S_{i}^{\text {down }}$ and $S_{j}^{\text {down }}$ by adding edges $K_{(0,24 i)} K_{\left(12 N^{2}-1,24 i\right)}, K_{\left(12 N^{2}-1,24 i\right)} K_{\left(-12 N^{2},-24 i-1\right)}, K_{\left(-12 N^{2},-24 i-1\right)} K_{(-t-1,-24 i-1)}$, $K_{(-t-1,-24 i-1)} K_{(-t-1,24 j)}, K_{(-t-1,24 j)} K_{(0,24 j)}$.

The remaining difficulties of the reduction are how to color the vertices of $G^{*}$ according to the requirements for Möbius DPZP and how to handle crossing paths. We should present techniques to handle them in the proof.

- Lemma 3.1. $m n$-DPZP is PPA-hard.

We conclude that Möbius DPZP and Möbius Tucker are PPA-complete. 


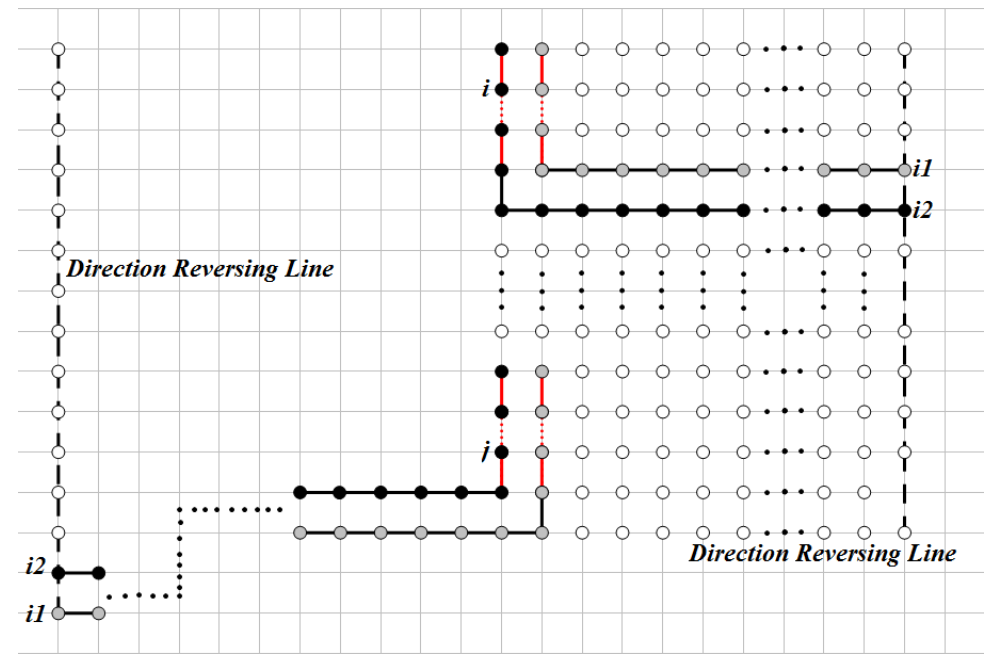

Figure 2 Connecting vertices 2 .

Theorem 3.2. $m n$-DPZP is PPA-complete.

Proof. By Lemma 2.14, mn-DPZP is in PPA. By Lemma 3.1, mn-DPZP is PPA-hard. The claim follows.

- Theorem 3.3. sm-Tucker is PPA-complete.

Proof. sm-Tucker is in PPA by Lemma 2.18.

For PPA-hardness, we use the same construction as the proof of Lemma 3.1, except that we change vertices colored 0 to color -2 . Therefore, at each vertex of color 0 in Lemma 3.1, we have an edge of color +2 and -2 ; and vice versa. The reduction follows.

Therefore, the theorem holds.

Finally we show that m-Sperner is PPA-complete.

- Theorem 3.4. m-Sperner is PPA-complete.

Proof. First, m-Sperner is in PPA by Lemma 2.16.

To prove it is PPA-hard, we simply replace vertices colored $\{-1,-2\}$ to color 0 in the instance constructed in the PPA-hardness proof of mn-DPZP. Finding a fully colored triangle $\delta$ in the m-Sperner instance will imply a true zero point in the mn-DPZP instance because the direction preserving condition, Definition 2.7, for mn-DPZP will prevent another vertex in the same base triangle of color $\in\{-1,-2\}$.

The claim follows.

\section{High Dimensional Non-orientable Discrete Fixed Point}

In the above, some 2D fixed point problems on the Möbius band are proven PPA-complete. The generalized problem in higher dimension space with all constant side lengths is considered in this section. The proof is motivated by a construction in [9]. To handle the non-orientable space, the key changes are on the snake lemma. We need a dicephalic snake version. Considerable changes and new ideas are required to make it through. To avoid tedious details, we should present a version of the construction and the proof. To observe the page limit, we place all the proofs and some lemmas at the appendices. 


\subsection{Uniform Boundary Discrete Fixed Points on Möbius Band}

We introduce a version here for which the boundary of the 2D Möbius band consists vertices all of the same color. Every instance of the problem has index 0 . This naturally leads to a version of the fixed point problem where one fixed point is given and another is sought after. We call such a case the uniform boundary coloring.

More precisely, the coloring function $f$ is of uniform boundary on Möbius band $B_{N, M}$ if it satisfies that: (1) $f((x, \pm M))=0, \forall x \in \mathbb{Z},-N \leq x \leq N$. (2) Möbius condition, i.e. $f((N, y))=f((-N,-y)), \forall y \in \mathbb{Z},-M \leq y \leq M$. Then the Möbius Sperner problem can be defined as follows.

- Definition 4.1 (Möbius Sperner). The input is a polynomial-time machine $F$ that generates a uniform boundary 3-coloring function $f$ on $B_{N, M}: F(\mathbf{p})=f(\mathbf{p}) \in\{0,1,2\}, \forall \mathbf{p} \in B_{N, M}$, as well as a panchromatic base triangle. The required output is another panchromatic base triangle on $B_{N, M}$.

Note that index $\left(B_{N, M}, f\right)$ is zero for a color function of uniform boundary on the Möbius band. According to Lemma 2.5, we have the following lemma:

- Lemma 4.2. For any uniform boundary 3-coloring of the triangulated Möbius band $B_{N, M}$, the number of panchromatic base triangles is even. Given one panchromatic base triangle, finding another is a PPA-complete problem.

\subsection{High Dimensional Möbius Sperner}

We extend the 2-dimensional uniform boundary Möbius Sperner proven PPA-complete in the above to higher dimension. First we define the well-behaved function.

- Definition 4.3 (Well-behaved Function [9]). A polynomial-time computable integer function $f$ is well-behaved, if $\exists n_{0}>0$ such that $\forall n \geq n_{0} 3 \leq f(n) \leq n / 2$.

Define $K_{\mathbf{p}}=\left\{\mathbf{q} \in \mathbb{Z}^{d} \mid q_{i}=p_{i}\right.$ or $\left.p_{i}+1, \forall 1 \leq i \leq d\right\}$.

For a positive integer $d$ and a vector $\mathbf{r} \in \mathbb{Z}_{+}^{d}$, let

$$
A_{\mathbf{r}}^{d}=\left\{\mathbf{p} \in \mathbb{Z}^{d} \mid-r_{i}+1 \leq p_{i} \leq r_{i}-1, \forall 1 \leq i \leq d\right\}
$$

be the hyper grid with side length $\mathbf{r}$ (note that is $2\left(r_{i}-1\right)$ in the $i$-th dimension because of symmetry with respect to $\left.r_{i}=0\right)$. Note that its boundary is, in one dimension, intentionally left open,

$$
\partial A_{\mathbf{r}}^{d}=\left\{\mathbf{p} \in A_{\mathbf{r}}^{d} \mid p_{i}=-r_{i}+1 \text { or } r_{i}-1, \exists 2 \leq i \leq d\right\}
$$

D Definition 4.4 (The Valid Boundary Condition). A coloring function $C: A_{\mathbf{r}}^{d} \rightarrow\{0,1, \ldots, d\}$ is valid on $A_{\mathbf{r}}^{d}$ if it satisfies the following Boundary Conditions:

1. (Uniform color boundary) For any $\mathbf{p} \in \partial A_{r}^{d}, C(\mathbf{p})=0$

2. (Reversing face consistency) $C\left(\left(r_{1}-1, x_{2}, x_{3}, \ldots, x_{d}\right)\right)=C\left(\left(-r_{1}+1,-x_{2}, x_{3}, \ldots, x_{d}\right)\right)$ for all $x_{i}$, where $i=2,3, \ldots, d$. Note that it is equivalent to merging $\left(r_{1}-1, x_{2}, x_{3}, \cdots, x_{d}\right)$ and $\left(-r_{1}+1,-x_{2}, x_{3}, \cdots, x_{d}\right)$ into one vertex.

The point set $\left\{\left( \pm\left(r_{1}-1\right), x_{2}, \ldots, x_{d}\right):-r_{i}<x_{i}<r_{i}, i=2,3, \ldots, d\right\}$ are called reversing face. Even though they are not on the boundary, we include (2) here to make sure the consistency of function values on the non-orientable space. Fixing other variables, $x_{3}, x_{4}, \cdots, x_{d}$, we have a reversing plane for the variables $x_{1}$ and $x_{2}$. 
For any well-behaved function $f$, we define a corresponding Möbius-Sperner fixed point problem as follows.

- Definition 4.5 (Möbius Sperner $^{f}$ ). For a well-behaved function $f$ and a parameter $n$, let $m=f(n)$ and $d=\lceil n / f(n)\rceil$. An input instance of Möbius Sperner ${ }^{f}$ is a pair $\left(C, 0^{n}\right)$ where $C$ is a valid coloring function with parameter $d$ and $\mathbf{r}$ where $r_{i}=2^{m}, \forall i: 1 \leq i \leq d$. Given a point $\mathbf{p} \in A_{\mathbf{r}}^{d}$ where $K_{\mathbf{p}}$ is of degree one, i.e., contains one panchromatic simplex in its triangulation, the output of this problem is another point $\mathbf{q} \neq \mathbf{p}$, such that $K_{\mathbf{q}}$ contains another panchromatic simplex.

We have the following theorem.

- Theorem 4.6. The problem Möbius Spernerf is PPA-complete for any well-behaved function $f$.

One can show that this problem is in PPA. To prove the hardness, similar to the orientable space [9], we embed an instance of Möbius Sperner ${ }^{f_{2}}$, known in PPA-complete, into one dimensional higher space iteratively till Möbius Sperner ${ }^{f}$. We should show that the process can be done in a polynomial number of state transformations. In Subsection B, we show three crucial lemmas for our reduction. In Subsection C, we employ these three lemmas iteratively to build up our construction. Please see the Appendix for the detail proofs.

\section{Discrete Fixed Points on Projective Space and Klein Bottle}

The results we have discussed above extend to other non-orientable spaces. The general idea is to slice out a Möbius band from the more complicated non-orientable space and to color it properly, then to patch the rest of the space. Two of the most interesting ones are the projective space and Klein Bottle. While the Möbius band can be embedded into 3D Euclidean space, neither the projective space nor the Klein bottle can. In this section, we make a reduction from DPZP to both the Möbius band and the projective plane for the PPA-hardness. As usually, as both cases are two dimensional objects, it is easy to triangulate them and to develop a path following algorithm.

We have discussed two types of discrete fixed point problems in the above. 1. finding one, and 2. (given one) finding another, dependent on the boundary conditions. As both the Projective space and the Klein bottle are closed without a boundary, we need to use the second version.

Our presentation will focus on the mn-DPZP version of the problems. The same applies to other types of discrete fixed point concepts discussed above. We omit them here as the results are similar.

- Theorem 5.1. Given a triangulated projective plane with vertices labelled $\{0, \pm 1, \pm 2\}$. It is PPA-complete to find another zero point.

- Theorem 5.2. Given a triangulated Klein bottle with vertices labelled $\{0, \pm 1, \pm 2\}$. It is PPA-complete to find another zero point.

\section{Remarks and Discussion}

We have discussed two types of discrete fixed point problems on the Möbius band: finding one, and (given one) finding another, dependent on the boundary conditions. We show both problems are PPA-complete for several versions of discrete fixed point models, including the Sperner's problem on the two dimensional Möbius band. 
Our first step focuses on the 2D version. We start with mn-DPZP, which finds a zero point of a discrete version of the continuous functions. Based on this result, we derive PPA-completeness proof of several other related fixed point problems on the Möbius band. We discuss finding another for Möbius Sperner and Index1-Brouwer on Möbius Band. We discuss finding one for sm-Tucker and mn-DPZP. They are switchable into the other types. For example, we can change all negative colored vertices to color 0 in mn-DPZP to obtain a "finding one" version for Möbius Sperner. We leave those cases out in this version and only exemplify useful structures and techniques choosing the most typical cases.

In this work, the link between non-orientable topological space and undirected path following computational paradigm, started by Grigni in [17], is further ratified by the simple structure of 2D Möbius band. It deepens our understanding of the computational complexity difference between the two classes PPAD and PPA in terms of the underlying topological structures.

The simplicity of our construction allows itself to extend beyond the 2D Möbius band to more general cases. For example, the PPA completeness of the finding another fixed point version extends naturally to the Klein Bottle, the projective space, and to other non-orientable surfaces [32]. Simplicity has played a role in raising further curiosities from the 2D Sperner work [7] in the orientable space, such as in $[25,16]$.

Further the results extend to higher dimensions, even for the case where each side is of a constant length. One such high dimension non-orientable space case of finding-another fixed point is presented in Section 4. The result extends to different related solution concepts as in the previous related concepts.

Note that the discrete fixed point problems in our discussion has an exponential size configuration. Otherwise, we can enumerate the space to find a solution by brute force. To compute colors and function values, a polynomial size circuit is given as an input. Alternatively, an oracle model returns those values in a unit oracle time [19]. It is known that there is an asymptotic matching bound for finding the Brouwer's fixed point in Euclidean space [8], which extends to other discrete fixed point models [11]. The same holds for the nonorientable space we discuss here. The lower bound holds simply because the problem is harder in the non-orientable space. The upper bound follows by the standard divide-and-conquer on the index adopted for the non-orientable space.

We would like to see the natural 2D Möbius Sperner will encourage more constructive works to develop a better knowledge of the PPA-complete class. In particular, as had suggested by Grigni [17], we would like to see the computational complexity of the Smith's Theorem, known in the class of PPA, be eventually resolved.

Acknowledgements. This work was partially supported by Tianyuan Special Funds of the National Natural Science Foundation of China (No. 11426026), the National Science Foundation of China (Grant No. 61173011) and a Project 985 grant of Shanghai Jiao Tong University. Qi's work was supported by the Research Grant Council of Hong Kong (ECS Project No. 26200314 and GRF Project No. 16213115).

We would like to acknowledge our indebtedness to Xi Chen and Christos H. Papadimitriou for helps, inspirations, suggestions and discussions on PPA problems. The great appreciation is also due to the anonymous reviewers. Their constructive criticism and suggestions have been incorporated in the current revision. 


\section{References}

1 James Aisenberg, Maria Luisa Bonet, and Sam Buss. 2-D Tucker is PPA complete. ECCC TR15-163, 2015.

2 Noga Alon. Combinatorial nullstellensatz. Combinatorics, Probability and Computing, 8, 1999.

3 Paul Beame, Stephen Cook, Jeff Edmonds, Russell Impagliazzo, and Toniann Pitassi. The relative complexity of NP search problems. Journal of Computer and System Sciences, 57(1):3-19, 1998. doi:10.1006/jcss.1998.1575.

4 Kathie Cameron. Thomason's algorithm for finding a second hamiltonian circuit through a given edge in a cubic graph is exponential on krawczyk's graphs. Discrete Mathematics, 235(1-3):69-77, 2001. Chech and Slovak 3. doi:10.1016/S0012-365X(00)00260-0.

5 Kathie Cameron and Jack Edmonds. Some graphic uses of an even number of odd nodes. Annales de l'institut Fourier, 49(3):815-827, 1999. URL: http://eudml.org/doc/75365.

6 Chih-Wei Chang, Ming Liu, Sunghyun Nam, Shuang Zhang, Yongmin Liu, Guy Bartal, and Xiang Zhang. Optical möbius symmetry in metamaterials. Phys. Rev. Lett., 105:235501, Dec 2010. doi:10.1103/PhysRevLett.105.235501.

7 Xi Chen and Xiaotie Deng. On the complexity of 2D discrete fixed point problem. In In Proceedings of the 33rd International Colloquium on Automata, Languages and Programming. Lecture Notes in Computer Science, pages 489-500. Springer-Verlag, 2006.

8 Xi Chen and Xiaotie Deng. Matching algorithmic bounds for finding a brouwer fixed point. $J A C M, 55(3), 2008$.

9 Xi Chen, Xiaotie Deng, and Shang-Hua Teng. Settling the complexity of computing two-player nash equilibria. J. ACM, 56(3):14:1-14:57, May 2009. doi:10.1145/1516512. 1516516.

10 C. Chevalley. Démonstration d'une hypothèse de m. artin. Abhandlungen aus dem Mathematischen Seminar der Universität Hamburg, 11(1):73-75, 1935. doi:10.1007/ BF02940714.

11 Xiaotie Deng, Qi Qi, Amin Saberi, and Jie Zhang. Discrete fixed points: Models, complexities, and applications. Mathematics of Operations Research, 36(4):636-652, 2011. doi:10.1287/moor.1110.0511.

12 Jack Edmonds and Laura Sanità. On finding another room-partitioning of the vertices. Electronic Notes in Discrete Mathematics, 36:1257-1264, 2010. doi:10.1016/j.endm. 2010. 05.159 .

13 M.C. Escher. Möbius strip ii (red ants). http://www.mcescher.com/Gallery/ recogn-bmp/LW441.jpg, 1963. [Online; accessed 3-March-2016].

14 Y. N. Fang, Yao Shen, Qing Ai, and C. P. Sun. Negative Refraction Induced by Möbius Topology. ArXiv e-prints, January 2015. URL: http://arxiv.org/abs/1501.05729.

15 Katalin Friedl, Gábor Ivanyos, Miklos Santha, and Yves F. Verhoeven. Locally 2dimensional sperner problems complete for the polynomial parity argument classes. submitted. In In Proceedings of the 6th Italian Conference on Algorithms and Complexity. Lecture Notes in Computer Science, pages 380-391. Springer-Verlag, 2006.

16 Paul Goldberg. The complexity of the path-following solutions of two-dimensional sperner/brouwer functions. arXiv:1506.04882 [cs.CC], 2015.

17 Michelangelo Grigni. A sperner lemma complete for PPA. Information Processing Letters, 77(5-6):255-259, 2001. doi:10.1016/S0020-0190(00)00152-6.

18 Dongran Han, Suchetan Pal, Yan Liu, and Hao Yan. Folding and cutting dna into reconfigurable topological nanostructures. Nat Nano, 5(10):712-717, 10 2010. doi: 10.1038/nnano.2010.193. 
19 Michael D Hirsch, Christos H Papadimitriou, and Stephen A Vavasis. Exponential lower bounds for finding brouwer fix points. Journal of Complexity, 5(4):379-416, 1989. doi: 10.1016/0885-064X (89)90017-4.

20 Takuya Iimura. A discrete fixed point theorem and its applications. Journal of Mathematical Economics, 39(7):725-742, 2003. doi:10.1016/S0304-4068(03)00007-7.

21 Emil Jerábek. Integer factoring and modular square roots. CoRR, abs/1207.5220, 2012. URL: http://arxiv.org/abs/1207.5220.

22 Shiva Kintali. A compendium of PPAD-complete problems. http://www.cs.princeton. edu/ kintali/ppad.html. [Online; accessed 3-March-2016].

23 Adam Krawczyk. The complexity of finding a second hamiltonian cycle in cubic graphs. Journal of Computer and System Sciences, 58(3):641-647, 1999. doi:10.1006/jcss. 1998. 1611.

24 O. L. Mangasarian. Equilibrium points of bimatrix games. Journal of the Society for Industrial and Applied Mathematics, 12(4):778-780, 1964. URL: http://www.jstor.org/ stable/2946349.

25 Ruta Mehta. Constant rank bimatrix games are ppad-hard. In Proceedings of the FourtySixth Annual ACM Symposium on Theory of Computing, STOC'14, pages 545-554, New York, NY, USA, 2014. ACM.

26 Dömötör Pálvölgyi. 2d-tucker is ppad-complete. In 5th International Workshop, WINE 2009, Rome, Italy, December 14-18, 2009. Proceedings, pages 569-574. Springer-Verlag, 2009.

27 Christos H. Papadimitriou. On the complexity of the parity argument and other inefficient proofs of existence. Journal of Computer and System Sciences, 48(3):498-532, 1994. doi: 10.1016/S0022-0000(05)80063-7.

28 Rahul Savani and Bernhard von Stengel. Hard-to-solve bimatrix games. Econometrica, 74(2):397-429, 2006. doi:10.1111/j.1468-0262.2006.00667.x.

29 Herbert E. Scarf. The approximation of fixed points of a continuous mapping. Cowles Foundation Discussion Papers 216R, Cowles Foundation for Research in Economics, Yale University, 1967. URL: http://EconPapers.repec.org/RePEc:cwl:cwldpp:216r.

30 A.G. Thomason. Hamiltonian cycles and uniquely edge colourable graphs. In B. Bollobás, editor, Advances in Graph Theory, volume 3 of Annals of Discrete Mathematics, pages 259-268. Elsevier, 1978. doi:10.1016/S0167-5060(08)70511-9.

31 Michael J Todd. The computation of fixed points and applications, volume 124. Springer Science \& Business Media, 2013.

32 Eric W. Weisstein. Klein bottle. http://mathworld.wolfram.com/KleinBottle.html. [Online; accessed 3-March-2016].

\section{A Omitted Figures and Proofs}

Proof of Lemma 2.13. First, a base triangle is index 1 if and only if its vertices are colored $\{x, 1,2\}$ where $x \notin\{1,2\}$. As all vertices in a base triangle are of distance 1 in $\infty$-metric. Therefore, $x$ can be neither -1 nor -2 by the direction preserving property. The only index 1 base triangle is $\{0,1,2\}$.

Next, as any internal base edge appears in the calculation of indices of two base triangles, the summation of the indices of them is either 0 or 2 , which equals to $0(\bmod 2)$. The claim follows.

Proof of Lemma 2.14. Since we have only one edge with $(2,1)$ on the boundary of the Möbius band, the index of edges along the boundary is 1 . Therefore, by Lemma 2.13, there 
is an odd number of zero point base triangles on the Möbius grid. Therefore, there is always a zero point inside the Möbius grid.

For the construction of the AEUL, we take the boundary edge $(2,1)$ as the origin vertex of AEUL. Two such edges of mn-DPZP are connected in AEUL if they are in the same base triangle. Any such edge in mn-DPZP is an leaf node in AEUL if it is the single $\{1,2\}$ edge in a base triangle.

Therefore, an end of lines of the AEUL instance is a base triangle of the mn-DPZP. Finding a zero point base triangle is an AEUL problem, and in PPA.

Proof of Lemma 3.1. Using the main structure presented above, we show how to color $B_{12 N^{2}, 24 N}$, so that for any zero point of this mn-DPZP, we can get a corresponding solution for the search problem AEUL.

The circuit $T_{n}$ of AEUL generates an undirected graph $G=\left(C_{n}, E\right)$, where $C_{n}=\{0,1\}^{n}$. An edge $(u, v)$ appears in $E$ iff $u \in T_{n}(v)$ and $v \in T_{n}(u)$.

So given any $G$, we construct an instance $\left(f, G^{*}\right)$ for mn-DPZP problem where $f$ is a coloring function for the generated $G^{*}=B_{12 N^{2}, 24 N}$. We should also use $T_{12 N^{2}, 24 N}$ to refer to $G^{*}$ in case of no ambiguity, with the understanding that $\left(-12 N^{2}, y\right)$ and $\left(12 N^{2},-y\right)$ are the same vertex.

In constructing the coloring function $f$ for $G^{*}$, we make use of the input circuit of $T_{n}$, to identify edges connecting a node to another, and vice versa, and to identify the degree one node of the AEUL graph.

We define the coloring function as follows:

1. Color vertices on the boundary according to the admissible conditions, Definition 2.9.

2. Color the long vertex tube: $\forall j:-24 N \leq j<12$, set $f((0, j))=2, f((1, j))=1$, which is a long vertex tube for the given degree one vertex $\mathbf{0}$.

3. Coat the long vertex tube (to protect positive colored 1 and 2 inside tube): $\forall j:-24 N \leq$ $j<12$, set $f((-1, j))=-1$ and $f((2, j))=-2$.

4. Color the other vertex tubes: $\forall i: 0<i<N$, set $f((0,24 i+k))=2, f((1,24 i+k))=$ $1, k=0,1,2, \ldots, 11$. We need to make some modifications in the colors for the case $k=0$ later.

5. Coat vertex tubes (to protect positive colored 1 and 2 inside tube): $\forall i: 0<i<N$ : $f((-1,24 i+k))=-1, f((2,24 i+k))=-2, k=0,1,2, \ldots, 11$.

6. Make feasible: fill in the the rest of the interior vertices by color -2 . Some of those vertices will be re-colorred in the following steps.

7. Direction preserving on end of lines: For a leaf vertex $i: 0<i<N$, we have $f(0,24 i)=$ $f(1,24 i)=0$.

8. Build an edge path: Given an edge $(i, j) \in E$, w.l.o.g., assume that $i<j$, we construct a path between $i$ and $j$ in $G^{*}$. Let $\left(i^{\prime}, j\right) \in E$ and $\left(i, j^{\prime}\right) \in E$. If $j>j^{\prime}$, then the upper end of tube for $i$ is connected to that of $j$, else the lower end of the tube for $i$ is connected to that of $j$. Therefore, there are four possibilities one end of the vertex tube is connected to another vertex tube.

a. $i>i^{\prime}$ and $j<j^{\prime}$ : Lower end of vertex tube for $i$ is connected to the upper end of the vertex tube for $j$. See Figure 1 .

b. $i<i^{\prime}$ and $j>j^{\prime}$ : Upper end of vertex tube for $i$ is connected to the lower end of the vertex tube for $j$. See Figure 1 .

c. $i<i^{\prime}$ and $j<j^{\prime}$ : Lower end of vertex tube for $i$ is connected to the lower end of the vertex tube for $j$. See Figure 2 .

d. $i>i^{\prime}$ and $j>j^{\prime}$ : Upper end of vertex tube for $i$ is connected to the upper end of the vertex tube for $j$. Similar to item (c). 


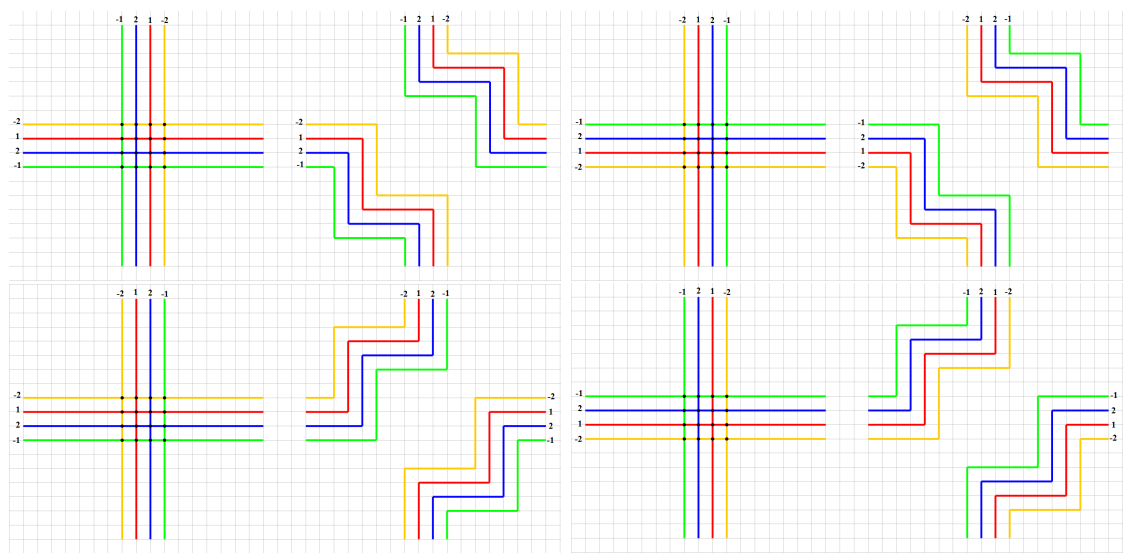

Figure 3 Connection Crossing: four cases are listed as Case 1, Case 2, Case 3, Case 4 as the normal order.

We should make appropriate adjustments so that the colorings consistently link two vertex tubes.

9. We need parallel paths of width 4 , making the colors crossing it to be $\langle-1,2,1,-2\rangle$ (or $\langle-2,1,2,-1\rangle$, dependent on the direction we are moving) to maintain the direction preserving conditions. The vertex tubes for $i$ and $j$ connected in the four ways specified above will maintain it, that their colorings are consistent.

Note that if the path will pass through the direction-reversing line, it must satisfy the Möbius condition, that is, the four vertices crossing the path reverse their colors from from $\langle-1,2,1,-2\rangle$ to $\langle-2,1,2,-1\rangle$ (or vice versa) after crossing the reversing direction boundary.

The colorings along the parallel paths satisfy our condition of direction preserving, as well as feasibility and admissibility conditions, except the problem where two paths cross each other. We resolve it in the same way originated from [7], shown in Figure 3. All the changes are local and can be decided using the local information with a constant bounded number of uses of the circuit $T$. Now we have provided the admissible coloring function that, given any point in $B_{12 N^{2}, 24 N}$, provides its coloring in polynomial time using the polynomial time circuit $T$.

Note that vertices of color 0 in $G^{*}$ only appear in the mapping from $G$ to $G^{*}$ from a vertex of degree one in $G$.

Therefore, finding a vertex of color 0 in $G^{*}$ is equivalent to find the AEUL solution in $G$.

Hence we have proven that mn-DPZP is PPA-hard.

Proof of Lemma 4.2. Clearly, the degree of any instance is 0. Therefore, there is an even number of the fully colored base triangles. Given one fully colored Sperner base triangle, the existence of another follows by the above lemma.

The problem is in PPA because the relationship of two edges on a base triangle of colors $(1,2)$ still holds and the uniform color boundary condition prevents the paths in the underlying AEUL going out of boundary.

On the other direction, m-Sperner can be easily reduced to Uniform-Color-Boundary Möbius Sperner by coating an extra layer of vertices outside of the boundary and coloring them all zero. More specifically, for each instance of m-Sperner, we create a Uniform-ColorBoundary Möbius Sperner by adding new vertices $\{(i, \pm(M+1)):-N \leq i \leq N\}$ with all 

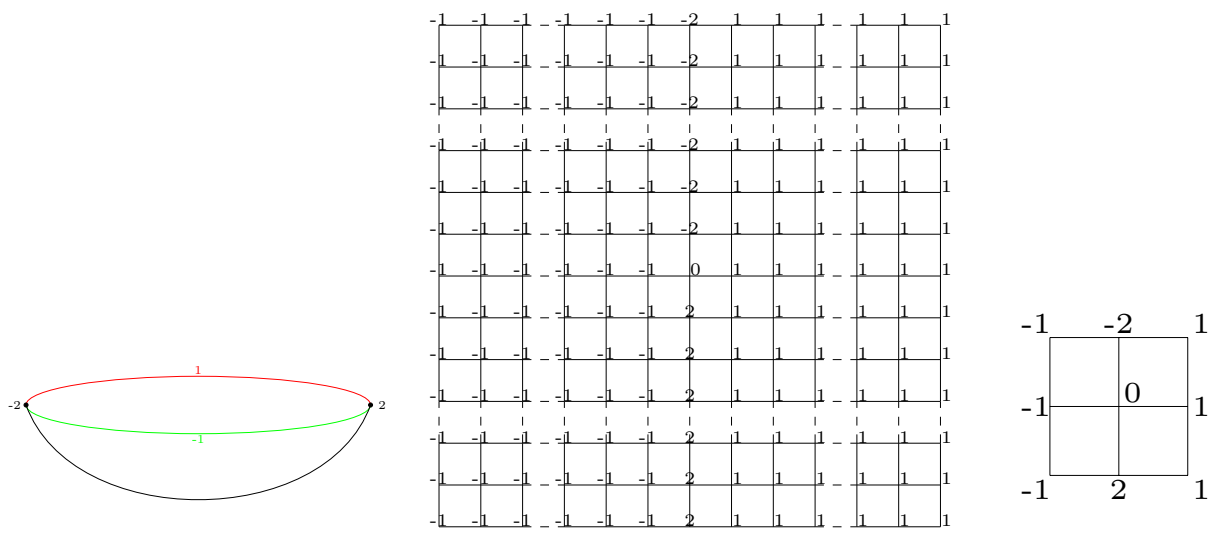

Figure 4 Disk.

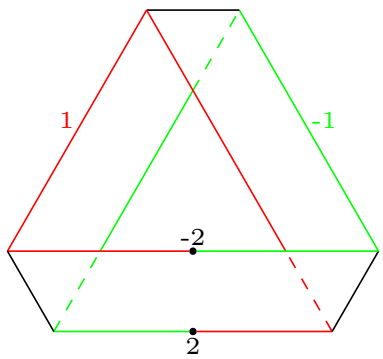

Figure 5 The Möbius Band.

color 0. After this construction, we have an instance of Uniform-Color-Boundary Möbius Sperner. There is a fully colored base triangle given $(\{(0,-M-1),(0,-M),(1,-M)\})$. Our goal is to find another which is also one for the original m-Sperner instance.

The Proof of Theorem 5.1. We make a reduction from mn-DPZP to the same problem on the projective space. We will define the DPZP on the projective plane. Then, we make a reduction of mn-DPZP to the DPZP on the projective plane.

First, a 2D projective plane can be obtained from the sphere of a $3 \mathrm{D}$ unit ball by identifying two points share the same diameter. In other words, $(x, y, z)$ and $(-x,-y,-z)$ in $B=\left\{(x, y, z): x^{2}+y^{2}+z^{2}=1\right\}$ are merged into one point.

Next, it can be decomposed into a Möbius band and a disc as follows. $M=\{(x, y, z):|z| \leq$ $1 / 2:(x, y, z) \in B\}$ and $D^{+}=\{(x, y, z) \in B: z \geq 1 / 2\} D^{-}=\{(x, y, z) \in B: z \leq-1 / 2\}$. Here $D^{+}$and $D^{-}$merge into one. We color the disc as in the central figure, at the center of the disk we place a zero point Figure 4 .

Further, let $M^{+}=\left\{(x, y, z) \in M: x \geq 0\right.$ and $M^{-}=\{(x, y, z) \in M: x \leq 0$. We have $M^{+}$is a Möbius band and so is $M^{-}$. Moreover, the interior of $M^{-}$maps into that of $M^{+}$in a 1-1 mapping. They have a shared boundary on $x=0$ that corresponds to the direction reversing line discussed in the above. Using the standard mn-DPZP boundary condition 2.9, we can embed an mn-DPZP instance on $M^{+}$as in Figure 5.

Connecting the Möbius band on $\mathrm{M}^{+}$with the disk $D^{+}$along their boundaries, we construct a triangulated projective plane that has a zero point on $D^{+}$with the task of finding another zero point, which can only be on $M^{+}$. As $M^{+}$is equivalent to an mn-DPZP instance, it follows that the task is a PPA-complete problem. 

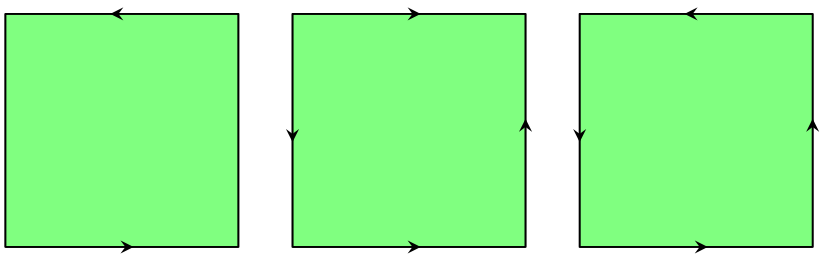

Figure 6 Grid Views: Möbius band, Klein bottle and Projective plane.

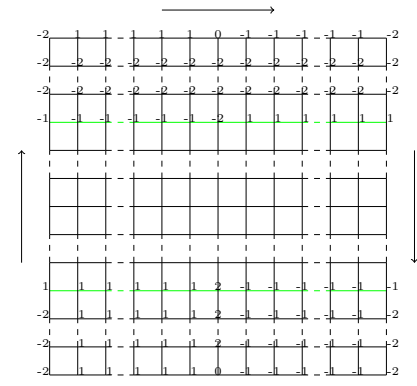

Figure 7 Embed DPZP on to Klein bottle.

The Proof of Theorem 5.2. Similarly, the PPA completeness of the finding another fixed point version extends naturally to the Klein Bottle [32]. Here again, the Klein Bottle can only be embedded in the four dimensional space. It is rather awkward to present it in the 3D world we live. Here we present by a $2 \mathrm{D}$ view with some amendments for ease of discussion in Figure 6. All the three non-orientable 2D spaces are represented uniformly in the 2D grid, with their boundaries merged with the opposite sides as illustrated.

This clear presentation allows a simple embedment of the DPZP grid on to the Klein bottle as presented in the following Figure 7.

Here we merge the top line and the bottom line. On it, there is a zero point in the middle. We are asked to find another zero point on the Klein bottle constructed from this grid. If we remove the top 4 lines and the bottom four lines, we obtain a mn-DPZP on the Möbius band where the only other zero points could be hidden.

Therefore, given the top zero point, finding another is to find a zero point in the mn-DPZP for the Möbius band in the middle.

\section{B Three Technical Lemmas}

A triple $T=(C, d, \mathbf{r})$ is a coloring triple if $\mathbf{r} \in \mathbb{Z}^{d}$ with $r_{i} \geq 3$ for all $1 \leq i \leq d$ and $C$ is a valid coloring function with parameters $d$ and $\mathbf{r}$. Let Size $[C]$ denote the number of gates plus the number of input and output variables in a function $C$.

The embedding is carried out by a sequence of three polynomial-time transformations: $\mathbf{L}^{1}(T, t, u), \mathbf{L}^{2}(T, u)$, and $\mathbf{L}^{3}(T, t, a, b) . \mathbf{L}^{1}(T, t, u)$ increases the $t$-th dimension size of the hyper grid from $r_{t}$ to $u$ (requiring $\left.u>r_{t}\right)$. $\mathbf{L}^{2}(T, u)$ extend the colouring into a space one dimension higher. $\mathbf{L}^{3}(T, t, a, b)$ folds a Möbius grid $T$ to $T^{\prime}$ so that one more side length in a dimension is reduced to a constant size. At the same time, from every panchromatic simplex of $T^{\prime}$, one can find a panchromatic simplex of $T$ efficiently. We should use $\mathbf{e}_{i}$ as the vector for the $i$-coordinate. 
$\mathbf{L}^{1}(T, t, u):\{$ Input: $T=(C, d, \mathbf{r}), t, u\}\left\{\right.$ Output: $\left.\left(C^{\prime}, d, \mathbf{r}^{\prime}\right), \mathbf{r}^{\prime}=\mathbf{r}+\left(u-r_{t}\right) \mathbf{e}_{t}\right\}$

1. if $\mathbf{p} \in \partial A_{\mathbf{r}^{\prime}}^{d}$ then $C^{\prime}(\mathbf{p})=0$

2. else if $-r_{t}<p_{t}<r_{t}$ then $C^{\prime}(\mathbf{p})=C(\mathbf{p})$

3. else $C^{\prime}(\mathbf{p})=0$

Figure 8 How $\mathbf{L}^{1}(T, t, u)$ extends the coloring triple $T=(C, d, \mathbf{r})$.

- Lemma $2.1\left(\mathbf{L}^{1}(T, t, u)\right.$ : Padding a Dimension). Given a coloring triple $T=(C, d, \mathbf{r})$ and two integers $1 \leq t \leq d$ and $u>r_{t}, \mathbf{L}_{1}$ constructs a new coloring triple $T^{\prime}=\left(C^{\prime}, d, \mathbf{r}^{\prime}\right)$ that satisfies the following two conditions:

(A) $r_{t}^{\prime}=u$, and $r_{i}^{\prime}=r_{i}$ for all other $i \in[d]$. In addition, there exists a polynomial $g_{1}(n)$ such that Size $\left[C^{\prime}\right]=$ Size $[C]+O\left(g_{1}\left(\operatorname{Size}\left[\mathbf{r}^{\prime}\right]\right)\right)$, and $T^{\prime}$ can be computed in time polynomial in Size $\left[C^{\prime}\right]$. We write $T^{\prime}=\mathbf{L}^{1}(T, t, u)$;

(B) From each panchromatic simplex $P^{\prime}$ of coloring triple $T^{\prime}$, we can compute a panchromatic simplex $P$ of $T$ in polynomial time.

Proof. Property A immediately follows from Figure 8. For Property $\mathbf{B}$, let $P^{\prime}$ be a panchromatic simplex of $T^{\prime}$, and $K_{\mathbf{p}}$ be the hypercube containing $P^{\prime}$. We first note that $-r_{t}+1 \leq p_{t}<r_{t}-1$, because if $p_{t} \geq r_{t}-1$ or $p_{t}<-r_{t}+1$, all colors on $K_{\mathbf{p}}$ will be 0 by the color assignment. As $C^{\prime}(\mathbf{q})=C(\mathbf{q})$ for all $\mathbf{q} \in A_{\mathbf{r}}^{d}$. Thus $P^{\prime}$ is also a panchromatic simplex of the coloring triple $T$.

Next, we add a dimension to the grid.

- Lemma $2.2\left(\mathbf{L}^{2}(T, u)\right.$ : Adding a Dimension). Given a coloring triple $T=(C, d, \mathbf{r})$ and integer $u \geq 3, \mathbf{L}^{2}$ constructs a new coloring triple $T^{\prime}=\left(C^{\prime}, d+1, \mathbf{r}^{\prime}\right)$ satisfying the following conditions:

(A) $r_{d+1}^{\prime}=u$, and $r_{i}^{\prime}=r_{i}$ for all $i \in[d]$. Moreover, there exists a polynomial $g_{2}(n)$ such that Size $\left[C^{\prime}\right]=$ Size $[C]+O\left(g_{2}\left(\operatorname{Size}\left[\mathbf{r}^{\prime}\right]\right)\right)$. $T^{\prime}$ can be computed in time polynomial in Size $\left[C^{\prime}\right]$. We write $T^{\prime}=\mathbf{L}^{2}(T, u)$;

(B) From each panchromatic simplex $P^{\prime}$ of coloring triple $T^{\prime}$, we can compute a panchromatic simplex $P$ of $T$ in polynomial time.

Proof. For each point $\mathbf{p} \in A_{\mathbf{r}^{\prime}}^{d+1}$, we use $\widehat{\mathbf{p}}$ to denote the point $\mathbf{z} \in A_{\mathbf{r}}^{d}$ with $z_{i}=p_{i}, \forall i \in[d]$. The color assignment of $C^{\prime}$ is given in Figure 9. Clearly, Property $\mathbf{A}$ is true.

To prove Property $\mathbf{B}$, we let $P^{\prime} \subset K_{p}$ be a panchromatic simplex of $T^{\prime}$. We note that $p_{d+1}=0$. For otherwise, $K_{p}$ contains color $d+1$ only if $p_{d+1}=-1$, in which case, it only contains color $d+1$ and 0 , a contradiction. Therefore, the panchromatic simplex $P^{\prime}$ must be in $K_{p}$ for $p_{d+1}=0$. The rest of vertices, those in $\hat{\mathbf{p}}$, must all be in $K_{p}$, which contains all the colors except $d+1$, is therefore a panchromatic simplex of $T$.

Lemma $2.3\left(\mathbf{L}^{3}(T, t, a, b)\right.$ : Dicephalic Snake Embedding). Given a coloring triple $T=$ $(C, d, \mathbf{r})$ and integer $1 \leq t \leq d$, if $r_{t}=a(2 b+1)+5$ for two integers $a, b \geq 1$, then $\mathbf{L}^{3}$ constructs a new coloring triple $T^{\prime}=\left(C^{\prime}, d+1, \mathbf{r}^{\prime}\right)$ that satisfies the following conditions:

(A) $r_{t}^{\prime}=a+5, r_{d+1}^{\prime}=4 b+3$, and $r_{i}^{\prime}=r_{i}$ for all other $i \in[d]$. Moreover, there exists a polynomial $g_{3}(n)$ such that Size $\left[C^{\prime}\right]=$ Size $[C]+O\left(g_{3}\left(\operatorname{Size}\left[\mathbf{r}^{\prime}\right]\right)\right)$ and $T^{\prime}$ can be computed in time polynomial in Size $\left[C^{\prime}\right]$. We write $T^{\prime}=\mathbf{L}^{3}(T, t, a, b)$.

(B) From each panchromatic simplex $P^{\prime}$ of coloring triple $T^{\prime}$, we can compute a panchromatic simplex $P$ of $T$ in polynomial time. 
$\mathbf{L}^{2}(T, u):\{$ Input: $T=(C, d, \mathbf{r}), u\}\left\{\right.$ Output: $T^{\prime}=\left(C^{\prime}, d+1, \mathbf{r}^{\prime}\right), r_{d+1}^{\prime}=u,(\forall i: 1 \leq i \leq$ d) $\left.r_{i}^{\prime}=r_{i}\right\}$

1. if $\mathbf{p} \in \partial A_{r^{\prime}}^{d+1}$ then $C^{\prime}(\mathbf{p})=0$.

2. else if $p_{d+1}=1$ then $C^{\prime}(\mathbf{p})=C(\widehat{\mathbf{p}})$ where $\widehat{\mathbf{p}} \in \mathbb{Z}^{d}$ satisfying $\widehat{p_{i}}=p_{i}$ for all $1 \leq i \leq d$.

3. else if $p_{d+1}=0$ then $C^{\prime}(\mathbf{p})=d+1$.

4. else $C^{\prime}(\mathbf{p})=0$

Figure 9 How $\mathbf{L}^{2}(T, u)$ extends the coloring triple $T=(C, d, \mathbf{r})$.

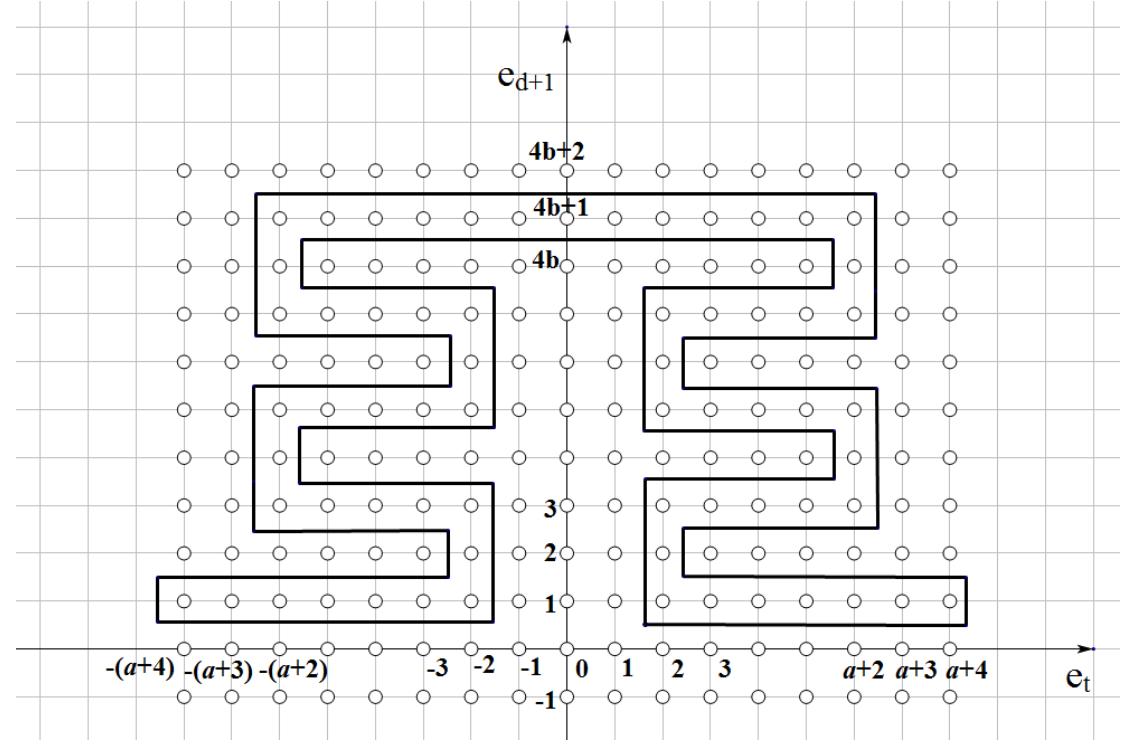

Figure 10 The two dimensional view of set $W \subset A_{\mathbf{r}^{\prime}}^{d+1}$.

Proof. Consider the domains $A_{\mathbf{r}}^{d} \subset \mathbb{Z}^{d}$ and $A_{\mathbf{r}^{\prime}}^{d+1} \subset \mathbb{Z}^{d+1}$ of our coloring triples. The reduction $\mathbf{L}^{3}(T, t, a, b)$ is carried out in three steps. First, we define a $d$-dimensional set $W \subset A_{\mathbf{r}^{\prime}}^{d+1}$ that is large enough to contain $A_{\mathbf{r}}^{d}$. Second, we define a (many to one) map $\psi$ from $W$ to $A_{\mathbf{r}}^{d}$ that specifies an implicit embedding of $A_{\mathbf{r}}^{d}$ into $W$. Finally, we build a function $C^{\prime}$ for $A_{\mathbf{r}^{\prime}}^{d+1}$ and show that from each panchromatic simplex of $T^{\prime}$, a panchromatic simplex of $T$ can be found in polynomial time.

A two dimensional view of $W \subset A_{\mathbf{r}^{\prime}}^{d+1}$ is illustrated in Figure 10. We use a (dicephalic) snake-pattern to realize the longer $t^{t h}$ dimension of $A_{\mathbf{r}}^{d}$ using the two-dimensional space defined by a new shorter $t^{\text {th }}$ dimension and the $(d+1)^{\text {th }}$ dimension (smaller by a multiplicative factor less than one) of $A_{\mathbf{r}^{\prime}}^{d+1}$, such that it is roughly $r_{t}=r_{t}^{\prime} * r_{d+1}^{\prime}$ (in fact, $r_{t}=O\left(r_{t}^{\prime} * r_{d+1}^{\prime}\right)$ ). Formally, $W$ consists of points $\mathbf{p} \in A_{\mathbf{r}^{\prime}}^{d+1}$ satisfying $1 \leq p_{d+1} \leq 4 b+1$ and

- if $p_{d+1}=1$, then $2 \leq p_{t} \leq a+4$ or $-(a+4) \leq p_{t} \leq-2$;

- if $p_{d+1}=4 b+1$, then $-(a+2) \leq p_{t} \leq a+2$;

- if $p_{d+1}=4(b-i)-1$ where $0 \leq i \leq b-1$, then $2 \leq p_{t} \leq a+2$ or $-(a+2) \leq p_{t} \leq-2$;

- if $p_{d+1}=4(b-i)-3$ where $0 \leq i \leq b-2$, then $2 \leq p_{t} \leq a+2$ or $-(a+2) \leq p_{t} \leq-2$;

- if $p_{d+1}=4(b-i)-2$ where $0 \leq i \leq b-1$, then $p_{t}=2$ or -2 ;

- if $p_{d+1}=4(b-i)$ where $0 \leq i \leq b-1$, then $p_{t}=a+2$ or $-(a+2)$.

To build $T^{\prime}$, we embed the coloring triple $T$ into $W$. The embedding is implicitly given by a many-to-one map $\psi$ from $W$ to $A_{\mathbf{r}}^{d}$, which will play a vital role in the coloring 
$\mathbf{L}^{3}(T, t, a, b):$

Input: $T=(C, d, \mathbf{r}), t, a, b, 1 \leq t \leq d, r_{t}=a(2 b+1)+5, a, b \geq 1$

Output: $T^{\prime}=\left(C^{\prime}, d+1, \mathbf{r}^{\prime}\right), r_{t}^{\prime}=a+5, r_{d+1}^{\prime}=4 b+3,(\forall i \neq t, 1 \leq i \leq d) r_{i}^{\prime}=r_{i}$

1. if $\mathbf{p} \in W$ then $C^{\prime}(\mathbf{p})=C(\psi(\mathbf{p}))$

2. else if $\mathbf{p} \in \partial A_{\mathbf{r}^{\prime}}^{d+1}$ then $C^{\prime}(\mathbf{p})=0$

3. else if $p_{d+1}=0$ then $C^{\prime}(\mathbf{p})=d+1$.

4. else if $p_{d+1}=4 i$ where $1 \leq i \leq b$ and $0 \leq\left|p_{t}\right| \leq a+1$ then $C^{\prime}(\mathbf{p})=d+1$

5. else if $p_{d+1}=4 i+1,4 i+2$ or $4 i+3$ where $0 \leq i \leq b-1$ and $\left|p_{t}\right| \leq 1$ then $C^{\prime}(\mathbf{p})=d+1$

6 . else $C^{\prime}(\mathbf{p})=0$

Figure 11 How $\mathbf{L}^{3}(T, t, a, b)$ extends the coloring triple $T=(f, d, \mathbf{r})$.

and the analysis of our reduction. For each $\mathbf{p} \in W$, we use $\mathbf{p}[m]$ to denote the point $\mathbf{q}$ in $\mathbb{Z}^{d}$ with $q_{t}=m$ and $q_{i}=p_{i}$ for all other $i \in[d]$. We denote by the function $\operatorname{sgn}(x)=1$ if $x>0,-1$ if $x<0,0$ if $x=0$. We define $\psi(\mathbf{p})$ according to the following cases:

- if $p_{d+1}=1$, then $\psi(\mathbf{p})=\mathbf{p}\left[2 a b \cdot \operatorname{sgn}\left(p_{t}\right)+p_{t}\right]$

- if $p_{d+1}=4 b+1$, then $\psi(\mathbf{p})=\mathbf{p}\left[p_{t}\right]$;

- if $p_{d+1}=4(b-i)-1$ where $0 \leq i \leq b-1$, then $\psi(\mathbf{p})=\mathbf{p}\left[((2 i+2) a+4) \cdot \operatorname{sgn}\left(p_{t}\right)-p_{t}\right]$;

- if $p_{d+1}=4(b-i)-3$ where $0 \leq i \leq b-2$, then $\psi(\mathbf{p})=\mathbf{p}\left[(2 i+2) a \cdot \operatorname{sgn}\left(p_{t}\right)+p_{t}\right]$;

- if $p_{d+1}=4(b-i)-2$ where $0 \leq i \leq b-1$, then $\psi(\mathbf{p})=\mathbf{p}\left[((2 i+2) a+2) \cdot \operatorname{sgn}\left(p_{t}\right)\right]$;

- if $p_{d+1}=4(b-i)$ where $0 \leq i \leq b-1$, then $\psi(\mathbf{p})=\mathbf{p}\left[((2 i+1) a+2) \cdot \operatorname{sgn}\left(p_{t}\right)\right]$.

We let $\psi_{i}(\mathbf{p})$ denote the $i^{\text {th }}$ component of $\psi(\mathbf{p})$.

- Proposition 2.4 (Valid Boundary Condition Preserving). The coloring function $C^{\prime}$ described in Figure 11 is valid on $A_{\mathbf{r}^{\prime}}^{d+1}$.

Proof. First we show that $C^{\prime}$ satisfies the uniform color boundary condition (1) for all $\mathbf{p} \in \partial A_{\mathbf{r}^{\prime}}^{d+1}$. We only need to prove every vertex $\mathbf{p} \in W \cap \partial A_{\mathbf{r}^{\prime}}^{d+1}$ is colored zero, by Step 2 of Figure 11 .

$\forall \mathbf{p} \in W \cap \partial A_{\mathbf{r}^{\prime}}^{d+1}$, by the definition of $\psi(\cdot)$, we have $p_{i}= \pm\left(r_{i}^{\prime}-1\right)$ if and only if $\psi_{i}(\mathbf{p})= \pm\left(r_{i}-1\right)$ for $(i: d \geq i \geq 2)$. It follows that $C^{\prime}(\mathbf{p})=C(\psi(\mathbf{p}))=0$ by the valid boundary condition for $C$. Therefore, $C^{\prime}$ satisfies the valid boundary condition (1).

Next we show that $C^{\prime}$ satisfies the reversing face boundary condition (2).

- If $t>2$, obviously, $C^{\prime}$ satisfies the boundary condition (2), since we have no change in $x_{1}$ nor $x_{2}$ for any set of other variables.

- If $t=1$, we consider $\mathbf{p}=\left(r_{1}^{\prime}-1, x_{2}, x_{3}, \ldots, x_{d}, x_{d+1}\right)$ and $\mathbf{p}^{\prime}=$ $\left(-r_{1}^{\prime}+1,-x_{2}, x_{3}, \ldots, x_{d}, x_{d+1}\right)$. If $x_{d+1} \neq 1, C^{\prime}(\mathbf{p})=C^{\prime}\left(\mathbf{p}^{\prime}\right)=0 . \quad$ If $x_{d+1}=1$, then $\mathbf{p}, \mathbf{p}^{\prime} \in W$. Thus $C^{\prime}(\mathbf{p})=C(\psi(\mathbf{p})), C^{\prime}\left(\mathbf{p}^{\prime}\right)=C\left(\psi\left(\mathbf{p}^{\prime}\right)\right)$. Since $C$ is valid, $\left.C(\psi(\mathbf{p}))=C\left(\psi\left(\mathbf{p}^{\prime}\right)\right)\right)$ by definition of $\psi(\cdot)$. Therefore, $C^{\prime}(\mathbf{p})=C^{\prime}\left(\mathbf{p}^{\prime}\right)$.

- If $t=2$, we consider $\mathbf{p}=\left(r_{1}-1, x_{2}^{\prime}, x_{3}, \ldots, x_{d}, x_{d+1}\right)$ and $\mathbf{p}^{\prime}=$ $\left(-r_{1}+1,-x_{2}^{\prime}, x_{3}, \ldots, x_{d}, x_{d+1}\right)$. Because $\mathbf{p}$ and $\mathbf{p}^{\prime}$ are central symmetric on the reversing plane, they are both in $W$ or both not. If $\mathbf{p}, \mathbf{p}^{\prime} \in W$, then $C^{\prime}(\mathbf{p})=C(\psi(\mathbf{p}))=C\left(\psi\left(\mathbf{p}^{\prime}\right)\right)=C^{\prime}\left(\mathbf{p}^{\prime}\right)$ (since $C$ is valid). If $\mathbf{p}, \mathbf{p}^{\prime}$ are not in $W$, we have $C^{\prime}(\mathbf{p})=C^{\prime}\left(\mathbf{p}^{\prime}\right)=0$ (where p is outside $W$ or $\left.p_{d+1}<0\right)$ or $C^{\prime}(\mathbf{p})=C^{\prime}\left(\mathbf{p}^{\prime}\right)=d+1$ (where $p$ is inside $W$ ).

Therefore, $C^{\prime}$ is a valid coloring function on $A_{\mathbf{r}^{\prime}}^{d+1}$. 
Clearly, whether $\mathbf{p} \in W$ or not can be decided in polynomial time by $\mathbf{L}^{3}$. Property $\mathbf{A}$ in Lemma 2.3 follows from the construction in Figure 11.

Next, we establish Property B of Lemma 2.3.

The intuition behind the proof is as follows. In $C^{\prime}$, vertices to the inside of $W$ are colored in $d+1$, and vertices to the outside are colored in 0. Every (unit-size) hypercube $K_{\mathbf{p}} \subset A_{\mathbf{r}^{\prime}}^{d+1}$ consists of $K_{\mathbf{p}} \cap W$, whose image $\psi\left(K_{\mathbf{p}} \cap W\right)$ is a (unit-size) hypercube in $A_{\mathbf{r}}^{d}$, and either vertices to the inside or the outside of $W$ but not both. Let $P^{\prime}$ be a panchromatic simplex of $T^{\prime}$ in $A_{\mathbf{r}^{\prime}}^{d+1}$. Let $K_{\mathbf{p}^{*}}$ be the hypercube containing $P^{\prime}$. Since hypercubes to the outside of $W$ do not have a vertex of color $d+1, K_{\mathbf{p}^{*}}$ must lie to the inside of $W$. We will show that, except the vertex of color $d+1$, every vertex $\mathbf{p} \in P^{\prime}$ either belongs to $W \cap K_{\mathbf{p}^{*}}$, or it can be mapped to a vertex $\mathbf{q} \in W \cap K_{\mathbf{p}^{*}}$, such that $C^{\prime}(\mathbf{q})=C^{\prime}(\mathbf{p})$. Thus from $P^{\prime}$, we can recover $d+1$ points in $W \cap K_{\mathbf{p}^{*}}$ with $d+1$ distinct colors $\{0,1, \ldots, d\}$. Since $C^{\prime}(\mathbf{p})=C(\psi(\mathbf{p}))$ for all $\mathbf{p} \in W$, we can apply $\psi$ to get a panchromatic simplex $P$ of $T$.

Formally, we proceed to prove a collection of claims to cover all the possible cases of the given panchromatic simplex $P^{\prime}$ of $T^{\prime}$. We use the following notation: For each $\mathbf{p} \in A_{\mathbf{r}^{\prime}}^{d+1}$, let $\mathbf{p}\left[m_{1}, m_{2}\right]$ denote the vertex $\mathbf{q} \subset \mathbb{Z}^{d+1}$ such that $q_{t}=m_{1}, q_{d+1}=m_{2}$ and $q_{i}=p_{i}$ for all other $i \in[d]$.

- Claim 2.5. If $p_{t}^{*}=0$, then $p_{d+1}^{*}=4 b$. Furthermore, for every vertex $\mathbf{p} \in P^{\prime}$ such that $C^{\prime}(\mathbf{p}) \neq d+1, C\left(\psi\left(\mathbf{p}\left[p_{t}, 4 b+1\right]\right)\right)=C^{\prime}(\mathbf{p})$.

Proof. For the first part of the claim, we have the following contradictions if $p_{d+1}^{*} \neq 4 b$ and $p_{t}^{*}=0$.

1. If $p_{d+1}^{*}=4 b+1, K_{\mathbf{p}^{*}}$ does not contain color $d+1$.

2. $p_{d+1}^{*}<0: C^{\prime}(\mathbf{p}) \in\{0, d+1\}$, the colors of vertices in $K_{\mathbf{p}^{*}}$ can only be 0 or $d+1$.

3. $p_{d+1}^{*}<4 b: p_{t}^{*}=0$ implies $p_{t} \in\{0,1\}$. Therefore, each vertex $\mathbf{q} \in K_{\mathbf{p}^{*}}$ is colored according one of the conditions in line $3,4,5$ or 6 of Figure 11. For each $\mathbf{q} \in K_{\mathbf{p}^{*}}, C^{\prime}(\mathbf{q})=0$ or $d+1$ from the construction in Figure 11.

Then, $K_{\mathbf{p}^{*}}$ cannot be a panchromatic hypercube, contradicting the assumption of the claim. Putting these cases together, we have $p_{d+1}^{*}=4 b$.

We now prove the second part of the claim. If $p_{d+1}=4 b+1$, then we are done, because $C(\psi(\mathbf{p}))=C^{\prime}(\mathbf{p})$ according to line 1 of Figure 11 . Then $p_{d+1}=4 b$ is the only other possibility. Therefore, by the condition $C^{\prime}(\mathbf{p}) \neq d+1$, according to Line 2 and 4 of Figure 11, we have $\mathbf{p} \in W \cap \partial A_{\mathbf{r}^{\prime}}^{d+1}$ and $\mathbf{p}\left[p_{t}, 4 b+1\right]=\mathbf{p}$. So we have $C\left(\psi\left(\mathbf{p}\left[p_{t}, 4 b+1\right]\right)\right)=C^{\prime}\left(\mathbf{p}\left[p_{t}, 4 b+1\right]\right)=$ $C^{\prime}(\mathbf{p})$, which completes the proof of the claim.

- Claim 2.6. If $p_{t}^{*}=a+2$ or $a+3$, then $p_{d+1}^{*}=0$. In addition, for each vertex $\mathbf{p} \in P^{\prime}$ such that $C^{\prime}(\mathbf{p}) \neq d+1, C\left(\psi\left(\mathbf{p}\left[p_{t}, 1\right]\right)\right)=C^{\prime}(\mathbf{p})$.

Proof. Obviously, $p_{d+1}^{*} \geq 0$. If $p_{d+1}^{*}>0$, then $K_{\mathbf{p}^{*}}$ does not contain color $d+1$, so we have $p_{d+1}^{*}=0$. The first half of the claim holds.

For the second half of the claim, first we know that if $\mathbf{p} \in W$, the claim follows. We consider the following three cases:

1. $p_{d+1}=1$ : then $\mathbf{p}$ is in $W$, we have $C\left(\psi\left(\mathbf{p}\left[p_{t}, 1\right]\right)\right)=C^{\prime}(\mathbf{p})$.

2. $\mathbf{p} \in A_{\mathbf{r}^{\prime}}^{d+1} \backslash \partial A_{\mathbf{r}^{\prime}}^{d+1}$ : recall that $C^{\prime}(\mathbf{q})=d+1$ for all $\mathbf{q} \in A_{\mathbf{r}^{\prime}}^{d+1} \backslash \partial A_{\mathbf{r}^{\prime}}^{d+1}$ with $q_{d+1}=0$, and we know that $p_{t} \in\{a+2, a+3, a+4\}$. So $\mathbf{p}$ is also in $W$ in this case.

3. $\mathbf{p} \in \partial A_{\mathbf{r}^{\prime}}^{d+1}$ : we have $\mathbf{p}\left[p_{t}, 1\right] \in \partial A_{\mathbf{r}^{\prime}}^{d+1}$, and $\psi(\mathbf{p})=\psi\left(\mathbf{p}\left[p_{t}, 1\right]\right)$, hence $C\left(\psi\left(\mathbf{p}\left[p_{t}, 1\right]\right)\right)=$ $C(\psi(\mathbf{p}))=C^{\prime}(\mathbf{p})$.

Combine these three cases, the second half of the claim follows. 
- Claim 2.7. If $p_{d+1}^{*}=4 b$, then $0 \leq p_{t}^{*} \leq a+1$. Moreover, for each vertex $\mathbf{p} \in P^{\prime}$ such that $C^{\prime}(\mathbf{p}) \neq d+1, C\left(\psi\left(\mathbf{p}\left[p_{t}, 4 b+1\right]\right)\right)=C^{\prime}(\mathbf{p})$.

Proof. If $p_{t}^{*}>a+1$, then $K_{\mathbf{p}^{*}}$ does not contain color $d+1$. So $0 \leq p_{t}^{*} \leq a+1$. Similar to the proof of Claim 2.5, we can prove the second part for the case when $0 \leq p_{t} \leq a+1$.

When $p_{t}=a+2$, both $\mathbf{p}$ and $\mathbf{p}\left[p_{t}, 4 b+1\right]$ are in $W$, and we have $\psi(\mathbf{p})=\psi\left(\mathbf{p}\left[p_{t}, 4 b+1\right]\right)$. Thus, $C\left(\psi\left(\mathbf{p}\left[p_{t}, 4 b+1\right]\right)\right)=C(\psi(\mathbf{p}))=C^{\prime}(\mathbf{p})$.

We can similarly prove the following claims.

- Claim 2.8. If $p_{d+1}^{*}=4 i+1$ or $4 i+2$ for some $0 \leq i \leq b-1$, then $p_{t}^{*}=1$. Moreover, for each $\mathbf{p} \in P^{\prime}$ such that $C^{\prime}(\mathbf{p}) \neq d+1, C\left(\psi\left(\mathbf{p}\left[2, p_{d+1}\right]\right)\right)=C^{\prime}(\mathbf{p})$.

- Claim 2.9. If $p_{d+1}^{*}=4 i$ for some $1 \leq i \leq b-1$, then $1 \leq p_{t}^{*} \leq a+1$. In addition, for each $\mathbf{p} \in P^{\prime}$ such that $C^{\prime}(\mathbf{p}) \neq d+1$, if $2 \leq p_{t} \leq a+1$, then $C\left(\psi\left(\mathbf{p}\left[p_{t}, 4 i+1\right]\right)\right)=C^{\prime}(\mathbf{p})$; if $p_{t}=1$, then $C(\psi(\mathbf{p}[2,4 i+1]))=C^{\prime}(\mathbf{p})$.

- Claim 2.10. If $p_{d+1}^{*}=4 i-1$ for some $1 \leq i \leq b$, then $1 \leq p_{t}^{*} \leq a+1$. Moreover, for each $\mathbf{p} \in P^{\prime}$ such that $C^{\prime}(\mathbf{p}) \neq d+1$, if $2 \leq p_{t} \leq a+1$, then $C\left(\psi\left(\mathbf{p}\left[p_{t}, 4 i-1\right]\right)\right)=C^{\prime}(\mathbf{p})$; if $p_{t}=1$, then $C(\psi(\mathbf{p}[2,4 i-1]))=C^{\prime}(\mathbf{p})$.

Claim 2.11. If $p_{d+1}^{*}=0$, then $1 \leq p_{t}^{*} \leq a+3$. In addition, for each vertex $\mathbf{p} \in P^{\prime}$ such that $C^{\prime}(\mathbf{p}) \neq d+1$, if $2 \leq p_{t}^{*} \leq a+3$, then $\mathbf{p} \in W$ (and thus, $\left.C(\psi(\mathbf{p}))=C^{\prime}(\mathbf{p})\right)$; if $p_{t}^{*}=1$, then $\left.C^{\prime} \psi(\mathbf{p}[2,1])\right)=C^{\prime}(\mathbf{p})$.

In addition,

Claim 2.12. $p_{d+1}^{*} \neq 4 b+1$.

Proof. If $p_{d+1}^{*}=4 b+1$ then $K_{\mathbf{p}^{*}}$ does not contain color $d+1$.

Here we do not list the cases where $p_{t}<0$ where they are all the same as the above claims since $W$ is symmetric (technically, there is one unit-sized bias between the negative and positive cases about the $t$ th dimension). Notice that $p_{d+1}^{*} \geq 0$ in our construction of $T^{\prime}$. Suppose that $P^{\prime}$ is a panchromatic simplex of $T^{\prime}$, and $K_{\mathbf{p}^{*}}$ be the hypercube containing $P^{\prime}$. Then $P^{\prime}$ and $\mathbf{p}^{*}$ must satisfy the conditions of one of the claims above. By that claim, we can transform every vertex $\mathbf{p} \in P^{\prime}$, (aside from the one that has color $d+1$ ) back to a vertex $\mathbf{q}$ in $A_{\mathbf{r}}^{d}$ to obtain a set $P$ from $P^{\prime}$. Since $P$ is accommodated, it is a panchromatic simplex of $t$. Thus, with all the claims above, we specify an efficient algorithm to compute a panchromatic simplex $P$ of $T$ given a panchromatic simplex $P^{\prime}$ of $T^{\prime}$.

\section{Proof of The PPA-hardness in Theorem 4.6}

Proof. Starting with the two dimensional case, a folding process presented next changes the size of each dimension one by one to make the size in accordance to that of the well-behaved functions. Each step uses operations $\mathbf{L}^{1}(T, t, u), \mathbf{L}^{2}(T, u)$, and $\mathbf{L}^{3}(T, t, a, b)$ to achieve this goal and maintains the validity of boundary conditions by Lemma 2.1, 2.2 and 2.3.

The folding process from Chen et al. [9] can now be copied over by using our versions of the three basic operations, $\mathbf{L}^{1}, \mathbf{L}^{2}$ and $\mathbf{L}^{3}$, introduced above. Only some little changes are necessary in order to deal with the details for the non-orientable model. We present a simplified version here. 


\section{The Construction of $T^{3 m^{\prime}-14}$ from $T^{1}$}

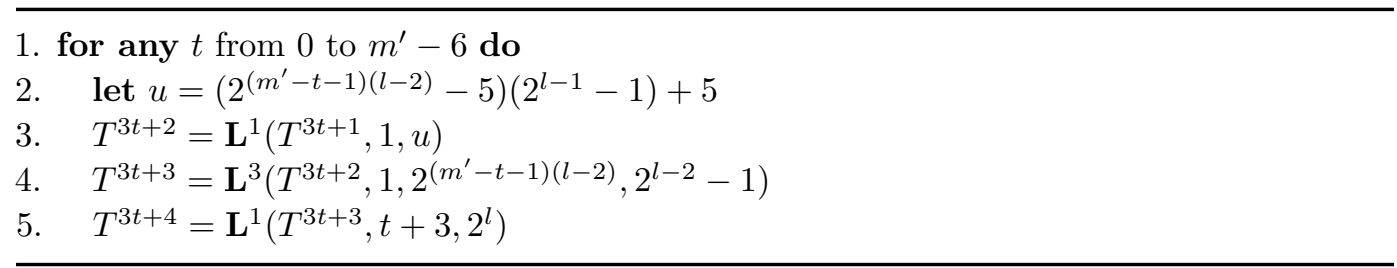

Figure 12 The Construction of $T^{3 m^{\prime}-14}$ from $T^{1}$.

\section{The Construction of $T^{w^{\prime}}$ from $T^{3 m^{\prime}-14}$}

1. let $t=0$

2. while $T^{3\left(m^{\prime}+t\right)-14}=\left(C^{3\left(m^{\prime}+t\right)-14}, m^{\prime}+t-3, \mathbf{r}^{3\left(m^{\prime}+t\right)-14}\right)$ satisfies $r_{1}^{3\left(m^{\prime}+t\right)-14}>2^{l}$ do

3. let $k=\left\lceil\left(r_{1}^{3\left(m^{\prime}+t\right)-14}-5\right) /\left(2^{l-1}-1\right)\right\rceil+5$

4. $\quad T^{3\left(m^{\prime}+t\right)-13}=\mathbf{L}^{1}\left(T^{3\left(m^{\prime}+t\right)-14}, 1,(k-5)\left(2^{l-1}-1\right)+5\right)$

5. $\quad T^{3\left(m^{\prime}+t\right)-12}=\mathbf{L}^{3}\left(T^{3\left(m^{\prime}+t\right)-13}, 1, k, 2^{l-2}-1\right)$

6. $\quad T^{3\left(m^{\prime}+t\right)-11}=\mathbf{L}^{1}\left(T^{3\left(m^{\prime}+t\right)-12}, m^{\prime}+t-2,2^{l}\right)$, set $t=t+1$

7. let $w^{\prime}=3\left(m^{\prime}+t\right)-13$ and $T^{w^{\prime}}=\mathbf{L}^{1}\left(T^{3\left(m^{\prime}+t\right)-14}, 1,2^{l}\right)$

Figure 13 The Construction of $T^{w^{\prime}}$ from $T^{3 m^{\prime}-14}$.

Formally, let $\left(C, 0^{2 n}\right)$ be an input instance of Möbius Sperner ${ }^{f_{2}}$, already proven PPAcomplete. Recall that $f_{2}(n)=\lfloor n / 2\rfloor$. Let

$$
l=f(11 n) \geq 3, m^{\prime}=\left\lceil\frac{n}{l-2}\right\rceil, \text { and } m=\left\lceil\frac{11 n}{l}\right\rceil .
$$

For any well-behaved function $f$, we reduce Möbius Sperner ${ }^{f_{2}}$ to Möbius Sperner ${ }^{f}$ by iteratively constructing a sequence of coloring triple $\mathcal{T}=\left\{T^{0}, T^{1}, \ldots, T^{w}\right\}$ for some $w=$ $O(m)$, where $T_{0}=\left(C, 2,\left(2^{n}, 2^{n}\right)\right)$ and $T_{w}=\left(C^{w}, m, \mathbf{r}^{w}\right)$ such that $\mathbf{r}^{w} \in \mathbb{Z}^{m}$ and $\mathbf{r}_{i}^{w}=2^{l}$ for any $i, 1 \leq i \leq m$. At each phase $t$, we employ one of the three technical lemmas $\mathbf{L}^{1}, \mathbf{L}^{2}$ and $\mathbf{L}^{3}$ described in the previous subsection with appropriate parameters to construct $T^{t+1}$ from $T^{t}$.

First, we invoke $\mathbf{L}^{1}\left(T^{0}, 1,2^{m^{\prime}(l-2)}\right)$ to get $T^{1}=\left(C^{1}, 2,\left(2^{m^{\prime}(l-2)}, 2^{n}\right)\right)$, where the precondition of $\mathbf{L}^{1}$ holds as $m^{\prime}(l-2) \geq n$. Next we call the procedure in Figure 12. During every loop, the first component of $\mathbf{r}$ decreases by a factor of $2^{l-2}$ while the dimension of the space increases by 1 and the new dimension has a size already satisfied the requirement. So when finishing this function, we get a temporary coloring triple $T^{3 m^{\prime}-14}=\left(C^{3 m^{\prime}-14}, d^{3 m^{\prime}-14}, \mathbf{r}^{3 m^{\prime}-14}\right)$, such that

$d^{3 m^{\prime}-14}=m^{\prime}-3, r_{1}^{3 m^{\prime}-14}=2^{5(l-2)}, r_{2}^{3 m^{\prime}-14}=2^{n}$ and $r_{i}^{3 m^{\prime}-14}=2^{l}$, for any $i: 3 \leq i \leq m^{\prime}-3$.

Next, well invoke the procedure given in Figure 13. Note that the while-loop must terminate in at most 8 iterations because we start with $r_{1}^{3 m^{\prime}-14}=2^{5(l-2)}$. The procedure returns a coloring triple $T^{w^{\prime}}=\left(C^{w^{\prime}}, d^{w^{\prime}}, \mathbf{r}^{w^{\prime}}\right)$ that satisfies

$$
w^{\prime} \leq 3 m^{\prime}+11, d^{w^{\prime}} \leq m^{\prime}+5, r_{1}^{w^{\prime}}=2^{l}, r_{2}^{w^{\prime}}=2^{n}, r_{i}^{w^{\prime}}=2^{l} \text {, for any } i: 3 \leq i \leq d^{w^{\prime}} .
$$


Then we repeat the whole process above on the second coordinate and obtain a coloring triple $T^{w^{\prime \prime}}=\left(C^{w^{\prime \prime}}, d^{w^{\prime \prime}}, \mathbf{r}^{w^{\prime \prime}}\right)$ such that

$$
w^{\prime \prime} \leq 6 m^{\prime}+21, d^{w^{\prime \prime}} \leq 2 m^{\prime}+8 \text { and } r_{i}^{w^{\prime \prime}}=2^{l} \text {, for any } i: 1 \leq i \leq d^{w^{\prime \prime}} .
$$

Now follow our initial definition for $m$ and $m^{\prime}$, we have

$$
d^{w^{\prime \prime}} \leq 2 m^{\prime}+8 \leq 2\left(\frac{n}{l-2}+1\right)+8 \leq 2\left(\frac{n}{l / 3}\right)+10=\frac{6 n}{l}+10 \leq \frac{11 n}{l} \leq m .
$$

Finally, we repeat applying $\mathbf{L}^{2}$ for $m-d^{w^{\prime \prime}}$ times with parameter $u=2^{l}$ to obtain the final coloring triple $T^{w}=\left(C^{w}\right), m, \mathbf{r}^{w}$ where $r_{i}^{w}=2^{l}$ for any $i, 1 \leq i \leq m$. It follows our construction, $w=O(m)$.

Now we prove that the whole construction is indeed a reduction from Möbius Sperner ${ }^{f_{2}}$ to Möbius Sperner ${ }^{f}$. Let $T^{i}=\left(C^{i}, d^{i}, \mathbf{r}^{i}\right)$, as sequence $\left\{\text { Size }\left[\mathbf{r}^{i}\right]\right\}_{0<i<w}$ is non-decreasing and $w=O(m)=O(n)$, by Property $\mathbf{A}$ of Lemma 2.1, 2.2 and 2.3, there exists a polynomial $g(n)$ such that Size $\left[C^{w}\right]=$ Size $[C]+O(g(n))$. By these Properties A again, we can construct the whole sequence $\mathcal{T}$ and in particularly, $T^{w}=\left(C^{w}, m, \mathbf{r}^{2}\right)$, in time polynomial in Size $[C]$.

As we know, the pair $\left(C^{w}, 0^{11 n}\right)$ is an input instance of Möbius Sperner ${ }^{f}$. Given a panchromatic simplex $P$ of $\left(C^{w}, 0^{11 n}\right)$, using the algorithm in Property $\mathbf{B}$ of Lemma 2.1, 2.2 and 2.3, we can compute a sequence of panchromatic simplex $P^{w}=P, P^{w-1}, \ldots, P^{0}$ iteratively in polynomial time, where $P^{t}$ is a panchromatic simplex of $T^{t}$ and can be computed from the panchromatic simplex $P^{t+1}$ of $T^{t+1}$. In the end, we obtain $P^{0}$, which is a panchromatic set of $\left(C, 0^{2 n}\right)$. 